IMA Journal of Numerical Analysis (2008) 28, 440-468

doi:10.1093/imanum/drm038

Advance Access publication on November 19, 2007

\title{
Interior penalty discontinuous Galerkin method for Maxwell's equations: optimal $L^{2}$-norm error estimates
}

\author{
Marcus J. GRote† AND ANNA SCHNEEBEli \\ Department of Mathematics, University of Basel, Rheinsprung 21, 4051 Basel, Switzerland \\ AND \\ DOMINIK SCHÖTZAU§ \\ Mathematics Department, University of British Columbia, 1984 Mathematics Road, \\ Vancouver, BC V6T 1Z2, Canada
}

[Received on 13 December 2006; revised on 10 September 2007]

\begin{abstract}
We consider the symmetric, interior penalty discontinuous Galerkin (DG) method for the time-dependent Maxwell's equations in second-order form. In Grote et al. (2007, J. Comput. Appl. Math., 204, 375 386), optimal a priori estimates in the DG energy norm were derived, either for smooth solutions on arbitrary meshes or for low-regularity (singular) solutions on conforming, affine meshes. Here, we show that the DG methods are also optimally convergent in the $L^{2}$-norm, on tetrahedral meshes and for smooth material coefficients. The theoretical convergence rates are validated by a series of numerical experiments in two-space dimensions, which also illustrate the usefulness of the interior penalty DG method for timedependent computational electromagnetics.
\end{abstract}

Keywords: Maxwell's equations; discontinuous Galerkin methods; a priori error estimates.

\section{Introduction}

With the need to simulate electromagnetic phenomena of increasing realism and complexity comes the need for more general numerical methods that easily handle complicated geometric features and different material properties. The first method for the numerical simulation of time-dependent electromagnetic waves, the finite-difference time domain (FDTD) scheme, was proposed by Yee (1966). Based on a finite-difference discretization of Maxwell's equations on two regular Cartesian grids, staggered both in space and in time, the FDTD method remains popular due to its simplicity and efficiency. However, like most finite-difference methods, the FDTD method is difficult to generalize to unstructured nonCartesian grids and suffers from the inaccurate representation of the solution on curved boundaries (staircase approximation; see Cangellaris \& Wright, 1991; Taflove, 1995). Moreover, its extension to higher order results in wider difference stencils, which require special treatment near physical boundaries.

In contrast, finite-element methods (FEMs) can handle unstructured grids and complex geometry; they easily extend to higher order, even in the vicinity of physical boundaries. They also provide rigorous a posteriori error estimates which are useful for local adaptivity and error control. Different finite-element discretizations of Maxwell's equations are available, such as the edge-element methods of

\footnotetext{
$\dagger$ Email: marcus.grote@unibas.ch.

†Email: anna.schneebeli@unibas.ch

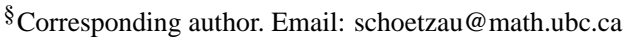

(c) The author 2007. Published by Oxford University Press on behalf of the Institute of Mathematics and its Applications. All rights reserved. 
Nédélec (1980, 1986), the node-based first-order formulation of Lee \& Madsen (1990), the node-based curl-curl formulation of Paulsen \& Lynch (1991) or the node-based least-squares FEM of Jiang et al. (1996) (see also Monk, 1992).

Two difficulties typically arise when standard (conforming) finite elements are used in practice. First, they are generally intended for use on a globally conforming mesh, i.e. a mesh without hanging nodes or mismatch of mesh points along internal boundaries. Unfortunately, the generation of highquality globally conforming meshes in arbitrary 3D geometry remains a formidable task, often done by hand, which can be more time consuming than the simulation itself. Second, although Nédélec element methods may be the most satisfactory from a theoretical point of view (see Monk, 2003), in particular near re-entrant corners, they are less attractive for time-dependent computations because the solution of a linear system is required at every time iteration. Indeed, in the case of triangular or tetrahedral edge elements, the entries of the diagonal matrix resulting from mass lumping are not necessarily strictly positive (see Elmkies \& Joly, 1997); therefore, explicit time stepping cannot be used in general. In contrast, nodal elements naturally lead to a fully explicit scheme when mass lumping is applied both in space and in time, but cannot correctly represent corner singularities in general.

Discontinuous Galerkin (DG) FEMs offer an attractive alternative to Nédélec's elements for the numerical solution of Maxwell's equations, in particular for time-dependent problems. Not only do they accommodate elements of various types and shapes, irregular nonmatching grids and even locally varying polynomial order, and hence offer great flexibility in the mesh design, but they also lead to (block) diagonal mass matrices and therefore yield fully explicit, inherently parallel methods when coupled with explicit time stepping. Indeed, the mass matrix arising from a DG discretization is always block diagonal, with block size equal to the number of degrees of freedom per element; hence, it can be inverted at very low computational cost. In fact, for constant material coefficients, the mass matrix is truly diagonal for a judicious choice of (locally orthogonal) shape functions. Because continuity across element interfaces is weakly enforced merely by adding suitable bilinear forms (the so-called numerical fluxes) to the standard variational formulation, the implementation of DG methods is straightforward within existing finite-element software libraries.

For the first-order hyperbolic systems, various DG FEMs are available. For instance, Cockburn \& Shu (1989) use a DG method in space combined with a Runge-Kutta (RK) scheme in time to discretize hyperbolic conservation laws; see also the survey article of Cockburn \& Shu (2001) and the references therein. In the work of Kopriva et al. (2000), DG methods are developed, which combine high-order spectral elements with a fourth-order low-storage RK scheme. A similar approach is used in the RK DG methods of Warburton (2000) and Hesthaven \& Warburton (2002), which combine high-order spatial accuracy with a fourth-order low-storage RK scheme. While successful, their schemes do not conserve energy due to upwinding. Fezoui et al. (2005) used central fluxes instead, yet the convergence rate of their scheme remains suboptimal. A stabilized central flux formulation was proposed in Hesthaven \& Warburton (2004) for the Maxwell eigenvalue problem, which yields additional control over spurious eigenmodes.

Recently, Chen et al. (2005) developed a high-order RKDG method for Maxwell's equations in first-order hyperbolic form, which achieves high-order convergence both in space and in time by using a strong stability-preserving (low-storage) RK scheme. By using locally divergence-free polynomials, Cockburn et al. (2004) developed a locally divergence-free DG method for the first-order Maxwell system. For the second-order (scalar) wave equation, Rivière \& Wheeler $(2001,2003)$ proposed a nonsymmetric formulation, which required additional stabilization for optimal convergence. A symmetric interior penalty DG method was first proposed by Grote et al. (2006), where optimal convergence rates in the energy norm and $L^{2}$-norm were shown; the usefulness of the method was also demonstrated via 
numerical experiments. Recently, Chung \& Engquist (2006) proposed a hybrid discontinuous/continuous finite-element approach for the acoustic wave equation.

In this paper, we continue and complete the work started in Grote et al. (2007) on symmetric interior penalty DG methods for the spatial discretization of Maxwell's equations in second-order form. Secondorder formulations indeed halve the number of unknowns and hence permit to achieve second- or higher order accuracy with the least amount of work and storage. They are also inherently time reversible, while both their semidiscrete and fully discrete approximations preserve (a discrete version of) the energy.

Our previous results (Grote et al., 2007) establish optimal a priori error estimates in a natural energy norm. Here, we shall show that the method also converges optimally in the $L^{2}$-norm (in space and time) on regular and shape-regular tetrahedral meshes and for smooth material coefficients. The proof of this result is based on suitable duality arguments and follows along the lines of the $L^{2}$-norm error analysis presented in Houston et al. (2005) for the time-harmonic Maxwell's equations. In fact, it heavily relies on some of the auxiliary technical lemmas presented there.

The outline of the paper is as follows: after stating the model problem in Section 2, we describe the interior penalty DG variational formulation in Section 3. In Section 4, we first review the error estimates of Grote et al. (2007), cf. Theorems 4.1 and 4.2, and then state our new $L^{2}$-norm error bound in Theorem 4.3. The proof of Theorem 4.3 is given in Section 5. In Section 6, numerical experiments in two-space dimensions illustrate the performance of our DG method and validate the theoretical error bounds. Finally, some concluding remarks are presented in Section 7.

\section{Model problem}

The evolution of a time-dependent electromagnetic field $\mathbf{E}(\mathbf{x}, t), \mathbf{H}(\mathbf{x}, t)$ propagating through a linear isotropic medium is determined by Maxwell's equations:

$$
\begin{aligned}
\varepsilon \mathbf{E}_{t} & =\nabla \times \mathbf{H}-\sigma \mathbf{E}+\mathbf{j}, \\
\mu \mathbf{H}_{t} & =-\nabla \times \mathbf{E} .
\end{aligned}
$$

Here, the coefficients $\mu, \varepsilon$ and $\sigma$ denote the relative magnetic permeability, the relative electric permittivity and the conductivity of the medium, respectively. The source term $\mathbf{j}$ corresponds to the applied current density. By eliminating the magnetic field $\mathbf{H}$, Maxwell's equations reduce to a second-order vector wave equation for the electric field $\mathbf{E}$ :

$$
\varepsilon \mathbf{E}_{t t}+\sigma \mathbf{E}_{t}+\nabla \times\left(\mu^{-1} \nabla \times \mathbf{E}\right)=\mathbf{j}_{t} .
$$

If the electric field is eliminated instead, one easily finds that the magnetic field $\mathbf{H}$ satisfies a similar vector wave equation, when both $\sigma$ and $\varepsilon$ are constant or $\sigma$ is identically zero.

Thus, we consider the following model problem: find the (electric or magnetic) field $\mathbf{u}(\mathbf{x}, t)$ such that

$$
\begin{aligned}
\varepsilon \mathbf{u}_{t t}+\sigma \mathbf{u}_{t}+\nabla \times\left(\mu^{-1} \nabla \times \mathbf{u}\right)=\mathbf{f} & \text { in } \Omega \times J, \\
\mathbf{n} \times \mathbf{u}=\mathbf{0} & \text { on } \Gamma \times J, \\
\left.\mathbf{u}\right|_{t=0}=\mathbf{u}_{0} & \text { in } \Omega, \\
\left.\mathbf{u}_{t}\right|_{t=0}=\mathbf{v}_{0} & \text { in } \Omega .
\end{aligned}
$$


Here, $J=(0, T)$ is a finite time interval and $\Omega$ is a bounded Lipschitz polyhedron in $\mathbb{R}^{3}$ with boundary $\Gamma=\partial \Omega$ and outward unit normal $\mathbf{n}$. For simplicity, we assume $\Omega$ to be simply connected and $\Gamma$ to be connected. The right-hand side $\mathbf{f}$ is a given source term in $L^{2}\left(J ; L^{2}(\Omega)^{3}\right)$.

Here, we denote by $L^{2}(\Omega)^{3}$ the Lebesgue space of square-integrable vector fields. The inner product and norm associated with this space are given by

$$
(\mathbf{u}, \mathbf{v})=\int_{\Omega} \mathbf{u} \cdot \mathbf{v} \mathrm{d} \mathbf{x} \quad \text { and } \quad\|\mathbf{u}\|_{0}=(\mathbf{u}, \mathbf{u})^{\frac{1}{2}} .
$$

The Bochner space $L^{2}\left(J ; L^{2}(\Omega)^{3}\right)$ then consists of time-dependent functions $\mathbf{u}(\mathbf{x}, t)$ such that

$$
\|\mathbf{u}\|_{L^{2}\left(J ; L^{2}(\Omega)^{3}\right)}=\left(\int_{J}\|\mathbf{u}(t)\|_{0}^{2} \mathrm{~d} t\right)^{\frac{1}{2}}<\infty,
$$

with $\mathbf{u}(t)$ being short-hand notation for the function $\mathbf{x} \mapsto \mathbf{u}(\mathbf{x}, t)$.

The functions $\mathbf{u}_{0}$ and $\mathbf{v}_{0}$ in (2.1) are prescribed initial data with $\mathbf{u}_{0} \in H_{0}(\operatorname{curl} ; \Omega)$ and $\mathbf{v}_{0} \in L^{2}(\Omega)^{3}$, where

$$
H_{0}(\operatorname{curl} ; \Omega)=\left\{\mathbf{v} \in L^{2}(\Omega)^{3}: \nabla \times \mathbf{v} \in L^{2}(\Omega)^{3}, \mathbf{n} \times \mathbf{v}=\mathbf{0} \text { on } \Gamma\right\} .
$$

Finally, we assume that the coefficients $\mu, \varepsilon$ and $\sigma$ are scalar positive functions that satisfy

$$
0<\mu_{\star} \leqslant \mu(\mathbf{x}) \leqslant \mu^{\star}<\infty, \quad 0<\varepsilon_{\star} \leqslant \varepsilon(\mathbf{x}) \leqslant \varepsilon^{\star}<\infty, \quad \mathbf{x} \in \Omega,
$$

and

$$
0 \leqslant \sigma(\mathbf{x}) \leqslant \sigma^{\star}<\infty, \quad \mathbf{x} \in \Omega,
$$

respectively. For simplicity, we also assume that $\mu$ is piecewise constant.

It follows from the results in Lions \& Magenes (1972) that problem (2.1) is well-posed and has a unique weak solution $\mathbf{u}(\mathbf{x}, t)$ with $\mathbf{u}(t) \in H_{0}(\operatorname{curl} ; \Omega)$ and $\mathbf{u}_{t}(t) \in L^{2}(\Omega)^{3}$ for all $t \in \bar{J}$.

\section{DG discretization}

\subsection{Meshes and finite-element spaces}

We consider meshes $\mathscr{T}_{h}$ that partition the domain into disjoint tetrahedral or hexahedral elements $\{K\}$, such that $\bar{\Omega}=\cup_{K \in \mathscr{T}_{h}} \bar{K}$. We assume that every element $K$ of the triangulation $\mathscr{T}$ is affine equivalent (see Section 2.3 of Ciarlet, 1978) to either a reference tetrahedron or a reference cube. We always assume that the partition is aligned with the discontinuities of the coefficient $\mu$.

For each $K \in \mathscr{T}_{h}$, we denote by $h_{K}$ the diameter of $K$ and by $\rho_{K}$ the diameter of the biggest ball included in $K$; as usual, the mesh size $h$ of $\mathscr{T}_{h}$ is given by $h=\max _{K \in \mathscr{T}_{h}} h_{K}$. We assume the meshes $\mathscr{T}_{h}$ to be shape regular. That is, they form a family $\left\{\mathscr{T}_{h}\right\}_{h}$ of triangulations such that

$$
\frac{h_{K}}{\rho_{K}} \leqslant \rho_{1} \quad \forall K \in \mathscr{T}_{h}, \forall h,
$$

with a constant $\rho_{1}>0$ that is independent of $K \in \mathscr{T}_{h}$ and the mesh size $h$. We allow for irregular meshes with hanging nodes, but assume that the local mesh sizes are of bounded variation. That is, there is a second constant $\rho_{2}>0$ such that

$$
\rho_{2} h_{K} \leqslant h_{K^{\prime}} \leqslant \rho_{2}^{-1} h_{K}
$$

for all neighbouring elements $K$ and $K^{\prime}$ in $\mathscr{T}_{h}$ and mesh sizes $h$. 
Finally, we call $\mathscr{T}_{h}$ regular if the intersection $\bar{K} \cap \bar{K}^{\prime}$ of two elements $K$ and $K^{\prime}$ is empty, a vertex, an entire edge or an entire face of both elements, i.e. the meshes do not contain irregular nodes. Clearly, regular and shape-regular meshes satisfy the bounded variation property (3.2).

We now introduce the finite-element space

$$
\mathbf{V}^{h}=\left\{\mathbf{v} \in L^{2}(\Omega)^{3}:\left.\mathbf{v}\right|_{K} \in \mathscr{S}^{\ell}(K)^{3}, K \in \mathscr{T}_{h}\right\},
$$

where $\mathscr{S}^{\ell}(K)$ is the space $\mathscr{P}^{\ell}(K)$ of polynomials of total degree at most $\ell$ on $K$ if $K$ is a tetrahedron and the space $\mathscr{Q}^{\ell}(K)$ of polynomials of degree at most $\ell$ in each variable on $K$ if $K$ is an affine hexadron.

\subsection{Trace operators}

Next, we define the trace operators needed for the DG discretization of the Maxwell operator (cf. Houston et al., 2004; Houston et al., 2005). To that end, let $\mathscr{T}_{h}$ be a triangulation of $\Omega$. An interior face $f=\partial K \cap \partial K^{\prime}$ is the (nonempty) intersection of the boundaries of two neighbouring elements $K$ and $K^{\prime}$ of $\mathscr{T}_{h}$. Similarly, a boundary face is given by $f=\partial K \cap \Gamma$ for a boundary element $K$. We denote by $\mathscr{F}_{h}^{\mathscr{I}}$ the set of all interior faces, by $\mathscr{F}_{h}^{\mathscr{B}}$ the set of all boundary faces and define $\mathscr{F}_{h}=\mathscr{F}_{h}^{\mathscr{I}} \cup \mathscr{F}_{h}^{\mathscr{B}}$.

For a piecewise smooth vector-valued function $\mathbf{u}$, we introduce the following trace operators. Let $f=\partial K \cap \partial K^{\prime} \in \mathscr{F}_{h}^{\mathscr{I}}$ be an interior face shared by the two elements $K^{+}$and $K^{-}$. We write $\mathbf{n}^{ \pm}$to denote the unit outward normal vectors on the boundaries $\partial K^{ \pm}$, respectively. Denoting by $\mathbf{u}^{ \pm}$ the traces of $\mathbf{u}$ taken from within $K^{ \pm}$, respectively, we define the tangential jumps and averages of $\mathbf{u}$ across $f$ by

$$
\llbracket \mathbf{u} \rrbracket_{\mathrm{T}}=\mathbf{n}^{+} \times \mathbf{u}^{+}+\mathbf{n}^{-} \times \mathbf{u}^{-}, \quad\{\mathbf{u}\}=\left(\mathbf{u}^{+}+\mathbf{u}^{-}\right) / 2,
$$

respectively. On a boundary face $f=\partial K \cap \Gamma \in \mathscr{F}_{h}^{\mathscr{B}}$, we set $\llbracket \mathbf{u} \rrbracket_{\mathrm{T}}=\mathbf{n} \times \mathbf{u}$ and $\{\mathbf{u}\}=\mathbf{u}$. Here, the trace of $\mathbf{u}$ is taken from within the boundary element $K$.

\subsection{DG semidiscretization}

For a given partition $\mathscr{T}_{h}$ of $\Omega$, an approximation order $\ell \geqslant 1$ and $t \in J$, we wish to approximate the exact solution $\mathbf{u}(\cdot, t)$ of (2.1) by a discrete function $\mathbf{u}^{h}(\cdot, t) \in \mathbf{V}^{h}$. Thus, we consider the following (semidiscrete) DG finite-element formulation: find $\mathbf{u}^{h}: \bar{J} \times V^{h} \rightarrow \mathbb{R}$ such that

$$
\begin{aligned}
\left(\varepsilon \mathbf{u}_{t t}^{h}, \mathbf{v}\right)+\left(\sigma \mathbf{u}_{t}^{h}, \mathbf{v}\right)+a_{h}\left(\mathbf{u}^{h}, \mathbf{v}\right) & =(\mathbf{f}, \mathbf{v}), \quad \mathbf{v} \in \mathbf{V}^{h}, \quad t \in J, \\
\left.\mathbf{u}^{h}\right|_{t=0} & =\Pi_{h} \mathbf{u}_{0}, \\
\left.\mathbf{u}_{t}^{h}\right|_{t=0} & =\Pi_{h} \mathbf{v}_{0} .
\end{aligned}
$$

Here, $\Pi_{h}$ is the $L^{2}$-projection onto $\mathbf{V}^{h}$. The discrete bilinear form $a_{h}$, defined on $\mathbf{V}^{h} \times \mathbf{V}^{h}$, is given by

$$
\begin{aligned}
a_{h}(\mathbf{u}, \mathbf{v})= & \sum_{K \in \mathscr{T}_{h}} \int_{K} \mu^{-1}(\nabla \times \mathbf{u}) \cdot(\nabla \times \mathbf{v}) \mathrm{d} \mathbf{x}-\sum_{f \in \mathscr{F}_{h}} \int_{f} \llbracket \mathbf{u} \rrbracket_{\mathrm{T}} \cdot\left\{\mu^{-1} \nabla \times \mathbf{v}\right\} \mathrm{d} A \\
& -\sum_{f \in \mathscr{F}_{h}} \int_{f} \llbracket \mathbf{v} \rrbracket_{\mathrm{T}} \cdot\left\{\mu^{-1} \nabla \times \mathbf{u}\right\} \mathrm{d} A+\sum_{f \in \mathscr{F}_{h}} \int_{f} \mathrm{a} \llbracket \mathbf{u} \rrbracket_{\mathrm{T}} \cdot \llbracket \mathbf{v} \|_{\mathrm{T}} \mathrm{d} A .
\end{aligned}
$$


The interior penalty function a penalizes the tangential jumps of $\mathbf{u}^{h}$ over the faces of the triangulation. To define it, we first introduce the functions $h$ and $m$ by

$$
\begin{aligned}
& \left.\mathrm{h}\right|_{f}= \begin{cases}\min \left\{h_{K}, h_{K^{\prime}}\right\}, & f \in \mathscr{F}_{h}^{\mathscr{I}}, f=\partial K \cap \partial K^{\prime}, \\
h_{K}, & f \in \mathscr{F}_{h}^{\mathscr{B}}, f=\partial K \cap \Gamma,\end{cases} \\
& \left.\mathrm{m}\right|_{f}= \begin{cases}\min \left\{\mu_{K}, \mu_{K^{\prime}}\right\}, & f \in \mathscr{F}_{h}^{\mathscr{I}}, f=\partial K \cap \partial K^{\prime}, \\
\mu_{K}, & f \in \mathscr{F}_{h} \mathscr{B}, f=\partial K \cap \Gamma .\end{cases}
\end{aligned}
$$

Here, $\mu_{K}$ is the restriction of the coefficient $\mu$ to element $K$. On each face $f \in \mathscr{F}_{h}$, we then set

$$
\left.\mathrm{a}\right|_{f}=\alpha \mathrm{m}^{-1} \mathrm{~h}^{-1},
$$

where $\alpha>0$ is chosen sufficiently large, independently of the mesh size and the magnetic permeability, see Lemma 3.1 below. This completes the semidiscrete formulation of the interior penalty DG method for the model problem in (2.1).

\subsection{Well-posedness}

To discuss the well-posedness of (3.4), we introduce the seminorm

$$
|\mathbf{v}|_{h}^{2}=\sum_{K \in \mathscr{T}_{h}}\left\|\mu^{-\frac{1}{2}}(\nabla \times \mathbf{v})\right\|_{0, K}^{2}+\sum_{f \in \mathscr{F}_{h}}\left\|\mathrm{a}^{\frac{1}{2}} \llbracket \mathbf{v}\right\|_{\mathrm{T}} \|_{0, f}^{2},
$$

with $\|\cdot\|_{0, K}$ and $\|\cdot\|_{0, f}$ denoting the $L^{2}$-norms over an element $K$ and a face $f$, respectively.

The following stability result holds (see Arnold et al., 2001, or Lemma 3.1 in Houston et al., 2004).

LEMMA 3.1 There is a threshold parameter $\alpha_{\min }>0$, independent of the mesh size and the permeability $\mu$, such that for $\alpha \geqslant \alpha_{\min }$,

$$
a_{h}(\mathbf{u}, \mathbf{u}) \geqslant C_{\text {coer }}|\mathbf{u}|_{h}^{2} \quad \forall \mathbf{u} \in \mathbf{V}^{h},
$$

with a coercivity constant $C_{\text {coer }}>0$ that is independent of the mesh size and the coefficient $\mu$.

The result in Lemma 3.1 implies that the discrete problem in (3.4) is well-posed and uniquely solvable provided that $\alpha \geqslant \alpha_{\min }$ (see, e.g. Arnold et al., 2001; Grote et al., 2006). We note that larger values of $\alpha$ result in a more restrictive CFL condition in (explicit) time discretizations of (3.4).

REMARK 3.2 When the interior penalty DG method is used for time-dependent computations, the finiteelement solution consists of a superposition of discrete eigenmodes. Because of symmetry, the energy of the semidiscrete formulation (3.4) is conserved, so that all the discrete modes neither grow nor decay in time. For eigenvalue computations, Buffa \& Perugia (2006) recently proved that the interior penalty DG discretization of the Maxwell operator is asymptotically free of spurious modes: the discrete spectrum will eventually converge to the continuous spectrum as $h \rightarrow 0$. Nonetheless, on any fixed mesh some of the discrete eigenmodes will not correspond to physical modes. Hesthaven \& Warburton (2002) and Warburton \& Embree (2006) showed that larger values of the penalty parameter in central flux or local discontinuous Galerkin (LDG) discretizations increase the separation between spurious and physical eigenmodes. Certainly as the mesh is refined, the energy present in the spurious modes will decrease and eventually vanish, as the numerical solution obtained with the interior penalty DG method converges to the exact solution (see Section 4). 


\section{A priori error bounds}

In this section, we state optimal a priori bounds for the error in the energy norm and the $L^{2}$-norm.

For a domain $D \subset \mathbb{R}^{3}$, we let $H^{\mathrm{s}}(D)$ be the Sobolev space of (possibly noninteger) order $s \geqslant 0$. The norm associated with this space is denoted by $\|\cdot\|_{s, D}$. Sobolev spaces of vector-valued functions are denoted by $H^{\mathrm{s}}(D)^{3}$. For simplicity, we write $\|\cdot\|_{s, D}$ for the product norm as well. If $D=\Omega$, we omit the dependence on the domain and simply write $\|\cdot\|_{s}$. Let $\mathbf{X}$ be a function space defined over $\Omega$, and let $\|\cdot\|_{\star}$ be its associated norm. For a time-dependent vector function $\mathbf{v}(\mathbf{x}, t)$, we define

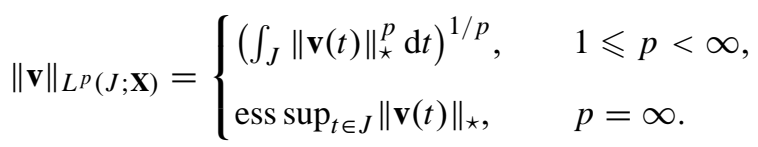

The corresponding Bochner space is $L^{p}(J ; \mathbf{X})=\left\{\mathbf{v}(\mathbf{x}, t):\|\mathbf{v}\|_{L^{p}(J ; \mathbf{X})}<\infty\right\}$.

\subsection{Energy norm error bounds}

We set $\mathbf{V}(h)=H_{0}(\operatorname{curl} ; \Omega)+\mathbf{V}^{h}$, and equip this space with the norm

$$
\|\mathbf{v}\|_{h}^{2}=\left\|\varepsilon^{\frac{1}{2}} \mathbf{v}\right\|_{0}^{2}+|\mathbf{v}|_{h}^{2}
$$

with $|\cdot|_{h}$ defined in (3.6).

The following error bound has been shown in Theorem 2 of Grote et al. (2007). It bounds the error in $L^{\infty}(J ; \mathbf{V}(h))$ and the time derivative of the error in $L^{\infty}\left(J ; L^{2}(\Omega)^{3}\right)$. This is an energy-type norm that is naturally associated with the discrete wave problem in (3.4). The error bound holds for shape-regular meshes of bounded variation consisting of tetrahedra and/or affine hexahedra (see (3.1) and (3.2)).

THEOREM 4.1 Let the meshes $\mathscr{T}_{h}$ be shape regular and of bounded variation. Let the solution $\mathbf{u}$ of (2.1) satisfy

$$
\mathbf{u} \in L^{\infty}\left(J ; H^{1+s}(\Omega)^{3}\right), \quad \mathbf{u}_{t} \in L^{\infty}\left(J ; H^{1+s}(\Omega)^{3}\right), \quad \mathbf{u}_{t t} \in L^{1}\left(J ; H^{\mathrm{s}}(\Omega)^{3}\right),
$$

for $s>\frac{1}{2}$. Let $\mathbf{u}^{h}$ be the semidiscrete DG approximation obtained with $\alpha \geqslant \alpha_{\min }$. Then, the error $\mathbf{e}=\mathbf{u}-\mathbf{u}^{h}$ satisfies

$$
\begin{aligned}
& \left\|\varepsilon^{\frac{1}{2}} \mathbf{e}_{t}\right\|_{L^{\infty}\left(J ; L^{2}(\Omega)^{3}\right)}+\|\mathbf{e}\|_{L^{\infty}(J ; \mathbf{V}(h))} \leqslant C\left(\left\|\varepsilon^{\frac{1}{2}} \mathbf{e}_{t}(0)\right\|_{0}+|\mathbf{e}(0)|_{h}\right) \\
& \quad+C h^{\min \{s, \ell\}}\left(\|\mathbf{u}\|_{L^{\infty}\left(J ; H^{1+s}(\Omega)^{3}\right)}+\left\|\mathbf{u}_{t}\right\|_{L^{\infty}\left(J ; H^{1+s}(\Omega)^{3}\right)}+\left\|\mathbf{u}_{t t}\right\|_{L^{1}\left(J ; H^{\mathrm{s}}(\Omega)^{3}\right)}\right),
\end{aligned}
$$

with a constant $C>0$ that is independent of the mesh size.

In Theorem 4.1, we implicitly assume that $\mathbf{u}_{0} \in H^{1+s}(\Omega)^{3}$ and $\mathbf{v}_{0} \in H^{\mathrm{s}}(\Omega)^{3}$. Hence, standard approximation properties of the $L^{2}$-projection imply that

$$
\left\|\varepsilon^{\frac{1}{2}} \mathbf{e}_{t}(0)\right\|_{0} \leqslant C h^{\min \{s, \ell+1\}}\left\|\mathbf{v}_{0}\right\|_{s}, \quad|\mathbf{e}(0)|_{h} \leqslant C h^{\min \{s, \ell\}}\left\|\mathbf{u}_{0}\right\|_{1+s} .
$$

As a consequence, Theorem 4.1 yields optimal convergence of order $\mathrm{O}\left(h^{\min \{s, l\}}\right)$ in the energy norm.

In many instances, solutions to the Maxwell's equations have singularities that do not satisfy the regularity assumptions in Theorem 4.1. Indeed, it is well-known that the strongest Maxwell singularities 
may have smoothness below $H^{1}(\Omega)^{3}$ (cf. Amrouche et al., 1998; Hiptmair, 2002; Monk, 2003). The following result from Theorem 3 of Grote et al. (2007) shows that DG methods converge in the energytype norm under weaker regularity assumptions, provided the meshes are regular and shape regular (consisting of tetrahedral and/or affine hexahedral elements). The restriction to regular meshes is due the use of curl-conforming Nédélec interpolants of the first kind in the proof.

THEOREM 4.2 Let the meshes $\mathscr{T}_{h}$ be regular and shape regular. Let the solution $\mathbf{u}$ of (2.1) satisfy

$$
\mathbf{u}, \mathbf{u}_{t}, \nabla \times \mathbf{u}, \nabla \times \mathbf{u}_{t} \in L^{\infty}\left(J ; H^{\mathrm{s}}(\Omega)^{3}\right)
$$

and

$$
\mathbf{u}_{t t}, \nabla \times \mathbf{u}_{t t} \in L^{1}\left(J ; H^{\mathrm{s}}(\Omega)^{3}\right),
$$

for $s>\frac{1}{2}$. Let $\mathbf{u}^{h}$ be the semidiscrete DG approximation obtained with $\alpha \geqslant \alpha_{\min }$. Then, the error $\mathbf{e}=\mathbf{u}-\mathbf{u}^{h}$ satisfies

$$
\begin{aligned}
\| \varepsilon^{\frac{1}{2}} \mathbf{e}_{t} & \left\|_{L^{\infty}\left(J ; L^{2}(\Omega)^{3}\right)}+\right\| \mathbf{e} \|_{L^{\infty}(J ; \mathbf{V}(h))} \\
\leqslant & C\left(\left\|\varepsilon^{\frac{1}{2}} \mathbf{e}_{t}(0)\right\|_{0}+|\mathbf{e}(0)|_{h}\right) \\
& +C h^{\min \{s, \ell\}}\left(\|\mathbf{u}\|_{L^{\infty}\left(J ; H^{\mathrm{s}}(\Omega)^{3}\right)}+\|\nabla \times \mathbf{u}\|_{L^{\infty}\left(J ; H^{\mathrm{s}}(\Omega)^{3}\right)}\right. \\
& +\left\|\mathbf{u}_{t}\right\|_{L^{\infty}\left(J ; H^{\mathrm{s}}(\Omega)^{3}\right)}+\left\|\nabla \times \mathbf{u}_{t}\right\|_{L^{\infty}\left(J ; H^{\mathrm{s}}(\Omega)^{3}\right)} \\
& \left.+\left\|\mathbf{u}_{t t}\right\|_{L^{1}\left(J ; H^{\mathrm{s}}(\Omega)^{3}\right)}+\left\|\nabla \times \mathbf{u}_{t t}\right\|_{L^{1}\left(J ; H^{\mathrm{s}}(\Omega)^{3}\right)}\right),
\end{aligned}
$$

with a constant $C>0$ that is independent of the mesh size.

If we additionally assume that $\mathbf{u}_{0} \in H^{1+s}(\Omega)^{3}$ for $s>0$, the bound in Theorem 4.2 yields again optimal convergence of the order $\mathrm{O}\left(h^{\min \{s, \ell\}}\right)$ for the error in the energy-type norm. For initial conditions with the lower regularity $\mathbf{u}_{0} \in H^{\mathrm{s}}(\Omega)^{3}$ and $\nabla \times \mathbf{u}_{0} \in H^{\mathrm{s}}(\Omega)^{3}, s>\frac{1}{2}$, we obtain the same result, provided the Nédélec projection is used to approximate the initial datum instead of the $L^{2}$-projection.

\section{2 $\quad L^{2}$-norm error bounds}

Theorems 4.1 and 4.2 immediately imply a (suboptimal) bound of order $\mathrm{O}\left(h^{\min \{s, \ell\}}\right)$ for the $L^{2}$-error, i.e. for $\left\|\mathbf{u}-\mathbf{u}^{h}\right\|_{L^{\infty}\left(J ; L^{2}(\Omega)^{3}\right)}$. We will now show that this estimate can be improved and that convergence of the optimal order $\mathrm{O}\left(h^{\ell+1}\right)$ can be obtained for smooth solutions and convex domains. For simplicity, we will assume that

$$
\mu \equiv 1, \quad \varepsilon \equiv 1,
$$

while no additional assumption on $\sigma$ is necessary. We remark that our proof immediately generalizes to smoothly varying $\mu$, but not to piecewise smooth $\mu$, because it is based on the duality techniques of Houston et al. (2005). In contrast to Houston et al. (2005), however, our proof also extends to arbitrary $\varepsilon$. We further note that our error estimate only holds on regular and shape-regular tetrahedral meshes. The same restriction on the underlying meshes appears in the conforming case where the Nédélec elements of the second kind are known to converge suboptimally in the $L^{2}$-norm (see Section 8.2.3 in Monk (2003) and Example 1 in Section 6 below). 
The $L^{2}$-error estimate depends on the regularity of the solution to the following problem:

Let $\mathbf{w} \in H_{0}(\operatorname{curl} ; \Omega)$ be divergence free and let $\mathbf{z}$ be the solution of the problem

$$
\begin{array}{rrr}
\nabla \times(\nabla \times \mathbf{z})+\mathbf{z}=\mathbf{w} & & \text { in } \Omega, \\
\mathbf{n} \times \mathbf{z}=\mathbf{0} & & \text { on } \Gamma .
\end{array}
$$

We conclude from Lemma 7.7 in Monk (2003) and the embedding results of Proposition 3.7 in Amrouche et al. (1998) that there is a parameter $\sigma_{E} \in\left(\frac{1}{2}, 1\right]$ and a stability constant $C_{\mathrm{S}}>0$ such that

$$
\mathbf{z} \in H^{\sigma_{E}}(\Omega)^{3}, \quad \nabla \times \mathbf{z} \in H^{\sigma_{E}}(\Omega)^{3}
$$

and

$$
\|\mathbf{z}\|_{\sigma_{E}}+\|\nabla \times \mathbf{z}\|_{\sigma_{E}} \leqslant C_{\mathrm{S}}\|\mathbf{w}\|_{0}
$$

The maximal value of $\sigma_{E}$ is closely related to the regularity properties of the Laplacian in polyhedra; in the case of constant coefficients considered here, it depends only on the opening angles at the corners and edges of the domain (cf. Amrouche et al., 1998). In particular, we have $\sigma_{E}=1$ for a convex domain.

THEOREM 4.3 Assume (4.1), and let the meshes $\mathscr{T}_{h}$ be regular and shape regular, and consist of tetrahedra. Let the solution $\mathbf{u}$ of (2.1) satisfy

$$
\begin{aligned}
& \mathbf{u} \in L^{\infty}\left(J, H^{\mathrm{s}+\sigma_{E}}(\Omega)^{3}\right), \quad \nabla \times \mathbf{u} \in L^{\infty}\left(J, H^{\mathrm{s}}(\Omega)^{3}\right), \\
& \mathbf{u}_{t} \in L^{\infty}\left(J, H^{\mathrm{s}+\sigma_{E}}(\Omega)^{3}\right), \quad \nabla \times \mathbf{u}_{t} \in L^{\infty}\left(J, H^{\mathrm{s}}(\Omega)^{3}\right),
\end{aligned}
$$

for $s>\frac{1}{2}$ and the regularity exponent $\sigma_{E} \in\left(\frac{1}{2}, 1\right]$ from (4.2). Let $\mathbf{u}^{h}$ be the semidiscrete DG approximation obtained on $\mathscr{T}_{h}$ with $\alpha \geqslant \alpha_{\min }$. Then, the error $\mathbf{e}=\mathbf{u}-\mathbf{u}^{h}$ satisfies

$$
\begin{aligned}
\|\mathbf{e}\|_{L^{\infty}\left(J ; L^{2}(\Omega)^{3}\right)} \leqslant & C h^{\min \{s, \ell\}+\sigma_{E}}\left(\|\mathbf{u}\|_{L^{\infty}\left(J ; H^{\left.s+\sigma_{E}(\Omega)^{3}\right)}\right.}+\|\nabla \times \mathbf{u}\|_{L^{\infty}\left(J ; H^{\mathrm{s}}(\Omega)^{3}\right)}\right. \\
& \left.+\left\|\mathbf{u}_{t}\right\|_{L^{\infty}\left(J ; H^{\mathrm{s}+\sigma_{E}}(\Omega)^{3}\right)}+\left\|\nabla \times \mathbf{u}_{t}\right\|_{L^{\infty}\left(J ; H^{\mathrm{s}}(\Omega)^{3}\right)}\right)
\end{aligned}
$$

with a constant $C>0$ that is independent of the mesh size.

For smooth solutions on convex domains $\left(\sigma_{E}=1\right)$, Theorem 4.3 thus yields optimal convergence in the $L^{2}$-norm:

$$
\|\mathbf{e}\|_{L^{\infty}\left(J ; L^{2}(\Omega)^{3}\right)} \leqslant C h^{\ell+1} .
$$

The proof of Theorem 4.3 will be given in Section 5 .

\section{Proof of $L^{2}$-estimate}

In this section, we present the proof of Theorem 4.3. The analysis follows the ideas used by Baker (1976) and Grote et al. (2006) for the scalar second-order wave equation. However, to overcome the additional difficulties caused by the Maxwell operator, we shall employ techniques similar to those developed by Houston et al. (2005) for the time-harmonic case. 


\subsection{Preliminaries}

We start by establishing and reviewing some auxiliary results. Recall that $\mu=\varepsilon=1$ (cf. the assumption in (4.1)).

5.1.1 Auxiliary form and error equation. As in Grote et al. (2007) and Houston et al. (2005), we introduce the auxiliary form $\widetilde{a}_{h}$ by setting

$$
\begin{aligned}
\widetilde{a}_{h}(\mathbf{u}, \mathbf{v})= & \sum_{K \in \mathscr{T}_{h}} \int_{K}(\nabla \times \mathbf{u}) \cdot(\nabla \times \mathbf{v}) \mathrm{d} \mathbf{x}-\sum_{f \in \mathscr{F}_{h}} \int_{f} \llbracket \mathbf{u} \rrbracket_{\mathrm{T}} \cdot\left\{\Pi_{h}(\nabla \times \mathbf{v})\right\} \mathrm{d} A \\
& -\sum_{f \in \mathscr{F}_{h}} \int_{f} \llbracket \mathbf{v} \rrbracket_{\mathrm{T}} \cdot\left\{\Pi_{h}(\nabla \times \mathbf{u})\right\} \mathrm{d} A+\sum_{f \in \mathscr{F}_{h}} \int_{f} \mathrm{a} \llbracket \mathbf{u} \rrbracket_{\mathrm{T}} \cdot \llbracket \mathbf{v} \rrbracket_{\mathrm{T}} \mathrm{d} A,
\end{aligned}
$$

where $\Pi_{h}$ is the $L^{2}$-projection onto $\mathbf{V}^{h}$. Clearly, the form $\widetilde{a}_{h}$ is well-defined over $\mathbf{V}(h) \times \mathbf{V}(h)$.

Since $a_{h}(\mathbf{u}, \mathbf{v})=\widetilde{a}_{h}(\mathbf{u}, \mathbf{v})$ for $\mathbf{u}, \mathbf{v} \in \mathbf{V}_{h}$, it follows immediately from Lemma 3.1 that, for $\alpha \geqslant \alpha_{\min }$,

$$
\widetilde{a}_{h}(\mathbf{u}, \mathbf{u}) \geqslant C_{\text {coer }}|\mathbf{u}|_{h}^{2}, \quad \mathbf{u} \in \mathbf{V}_{h}
$$

Furthermore, there exists a constant $C_{\text {cont }}>0$ independent of the mesh size and the coefficient $\mu$ such that

$$
\left|\widetilde{a}_{h}(\mathbf{u}, \mathbf{v})\right| \leqslant C_{\text {cont }}|\mathbf{u}|_{h}|\mathbf{v}|_{h}
$$

for all $\mathbf{u}, \mathbf{v} \in \mathbf{V}(h)$ (cf. Lemma 5 in Grote et al., 2007).

Next, for $\mathbf{u} \in H^{\mathrm{s}}(\Omega)^{3}$, with $\nabla \times \mathbf{u} \in H^{\mathrm{s}}(\Omega)^{3}$ for $s>\frac{1}{2}$, we define

$$
r_{h}(\mathbf{u} ; \mathbf{v})=\sum_{f \in \mathscr{F}_{h}} \int_{f} \llbracket \mathbf{v} \rrbracket_{\mathrm{T}} \cdot\left\{\nabla \times \mathbf{u}-\Pi_{h}(\nabla \times \mathbf{u})\right\} \mathrm{d} A,
$$

for any $\mathbf{v} \in \mathbf{V}(h)$. Obviously,

$$
r_{h}(\mathbf{u} ; \mathbf{v})=0 \quad \forall \mathbf{v} \in H_{0}(\operatorname{curl} ; \Omega)
$$

The following approximation result has been proved in Lemma 4.9 of Houston et al. (2005):

$$
\left|r_{h}(\mathbf{u} ; \mathbf{v})\right| \leqslant C_{\mathrm{R}} h^{\min \{s, \ell+1\}}|\mathbf{v}|_{h}\|\nabla \times \mathbf{u}\|_{s}, \quad \mathbf{v} \in \mathbf{V}(h),
$$

with a constant $C_{\mathrm{R}}>0$ that is independent of the mesh size.

Next, let $\mathbf{u}(\mathbf{x}, t)$ be the solution of the Maxwell's equation (2.1) and suppose that it satisfies the regularity assumption in Theorem 4.3. Let $\mathbf{u}^{h}$ be the semidiscrete DG approximation obtained with $\alpha \geqslant \alpha_{\min }$. Then, the error $\mathbf{e}=\mathbf{u}-\mathbf{u}^{h}$ satisfies

$$
\left(\mathbf{e}_{t t}, \mathbf{v}\right)+\left(\sigma \mathbf{e}_{t}, \mathbf{v}\right)+\widetilde{a}_{h}(\mathbf{e}, \mathbf{v})=r_{h}(\mathbf{u} ; \mathbf{v}), \quad \mathbf{v} \in \mathbf{V}^{h}, \text { a.e. in } J,
$$

see Grote et al. (2007). 
5.1.2 Nédélec space of the second kind. The largest conforming space underlying $\mathbf{V}^{h}$,

$$
\mathbf{V}^{c}=\mathbf{V}^{h} \cap H_{0}(\operatorname{curl} ; \Omega),
$$

is Nédélec's space of the second kind (see Nédélec, 1986, or Section 8.2 in Monk, 2003).

We denote by $\Pi_{\mathrm{N}}$ the Nédéléc interpolant of the second kind (see Nédélec, 1986). To review its approximation properties, let $s>\frac{1}{2}$ and consider $\mathbf{u} \in H_{0}(\operatorname{curl} ; \Omega) \cap H^{\mathrm{s}}(\Omega)^{3}$ with $\nabla \times \mathbf{u} \in H^{\mathrm{s}}(\Omega)^{3}$. Then, we have

$$
\begin{array}{r}
\left\|\mathbf{u}-\Pi_{\mathrm{N}} \mathbf{u}\right\|_{h} \leqslant C_{\mathrm{N}} h^{\min \{s, \ell\}}\left(\|\mathbf{u}\|_{s}+\|\nabla \times \mathbf{u}\|_{s}\right), \\
\left\|\nabla \times\left(\mathbf{u}-\Pi_{\mathrm{N}} \mathbf{u}\right)\right\|_{0} \leqslant C_{\mathrm{N}} h^{\min \{s, \ell\}}\|\nabla \times \mathbf{u}\|_{s},
\end{array}
$$

for a constant $C_{\mathrm{N}}>0$ that is independent of the mesh size. Additionally, if $s>0$ and $\mathbf{u} \in H_{0}(\operatorname{curl} ; \Omega) \cap$ $H^{1+s}(\Omega)^{3}$, then

$$
\left\|\mathbf{u}-\Pi_{\mathrm{N}} \mathbf{u}\right\|_{0} \leqslant C_{\mathrm{N}} h^{\min \{s, \ell\}+1}\|\mathbf{u}\|_{1+s} .
$$

A proof of the first two bounds in (5.8) can be found in Theorem 5.41, Remark 5.42 and Theorem 8.15 in Monk (2003). A proof of (5.9) has been given in Lemma 4.1 of Houston et al. (2005).

We further define the projection $\Pi^{c} \mathbf{u} \in \mathbf{V}^{c}=\mathbf{V}^{h} \cap H_{0}(\operatorname{curl} ; \Omega)$ by

$$
\left(\nabla \times\left(\mathbf{u}-\Pi^{c} \mathbf{u}\right), \nabla \times \mathbf{v}\right)+\left(\mathbf{u}-\Pi^{c} \mathbf{u}, \mathbf{v}\right)=0 \quad \forall \mathbf{v} \in \mathbf{V}^{c}
$$

An immediate consequence of this definition is that

$$
\left\|\mathbf{u}-\Pi^{c} \mathbf{u}\right\|_{h}=\inf _{\mathbf{v} \in \mathbf{V}^{c}}\|\mathbf{u}-\mathbf{v}\|_{h} .
$$

The approximation properties in (5.8) thus yield

$$
\left\|\mathbf{u}-\Pi^{c} \mathbf{u}\right\|_{h} \leqslant C_{\mathrm{N}} h^{\min \{s, \ell\}}\left(\|\mathbf{u}\|_{s}+\|\nabla \times \mathbf{u}\|_{s}\right) .
$$

The Nédélec space $\mathbf{V}^{c}$ in (5.7) can be decomposed into

$$
\mathbf{V}^{c}=\mathbf{X}^{h} \oplus \nabla S^{h},
$$

where

$$
\begin{aligned}
S^{h} & =\left\{q \in H_{0}^{1}(\Omega):\left.q\right|_{K} \in \mathscr{P}^{\ell+1}(K), K \in \mathscr{T}_{h}\right\}, \\
\mathbf{X}^{h} & =\left\{\mathbf{v} \in \mathbf{V}_{h}^{c}:(\mathbf{v}, \nabla q)=0 \forall q \in S^{h}\right\},
\end{aligned}
$$

respectively. The space $\mathbf{X}^{h}$ is referred to as the space of discretely divergence-free functions. By construction, the decomposition (5.12) is orthogonal in $L^{2}(\Omega)^{3}$ (cf. Section 8.2 in Monk, 2003).

The following approximation result can be established by proceeding as in Lemma 4.5 in Hiptmair (2002) and Lemma 7.6 in Monk (2003). For any $\mathbf{u} \in \mathbf{X}^{h}$, there is a divergence-free vector field $\mathbf{H u} \in$ $H_{0}(\operatorname{curl} ; \Omega)$ such that $\nabla \times \mathbf{H u}=\nabla \times \mathbf{u}$ and

$$
\|\mathbf{u}-\mathbf{H u}\|_{0} \leqslant C_{H} h^{\sigma_{E}}\|\nabla \times \mathbf{u}\|_{0},
$$


with $C_{H}>0$ independent of the mesh size and $\sigma_{E}$ denoting the parameter from (4.2). Moreover, we have

$$
\|\mathbf{H u}\|_{0} \leqslant\|\mathbf{u}\|_{0}
$$

Finally, we recall the approximation property of Proposition 4.5 in Houston et al. (2005) for discontinuous functions. For any $\mathbf{u} \in \mathbf{V}_{h}$, there is a function $\mathbf{u}^{c} \in \mathbf{V}^{c}$ such that

$$
\begin{aligned}
& \left\|\mathbf{u}-\mathbf{u}^{c}\right\|_{0} \leqslant C_{A}\left(\sum_{f \in \mathscr{F}_{h}} \int_{f} \mathrm{~h}\left|\llbracket \mathbf{u} \|_{\mathrm{T}}\right|^{2} \mathrm{~d} A\right)^{\frac{1}{2}}, \\
& \left\|\mathbf{u}-\mathbf{u}^{c}\right\|_{h} \leqslant C_{A}\left(\sum_{f \in \mathscr{F}_{h}} \int_{f} \mathrm{~h}^{-1}\left|\llbracket \mathbf{u} \|_{\mathrm{T}}\right|^{2} \mathrm{~d} A\right)^{\frac{1}{2}},
\end{aligned}
$$

with a constant $C_{A}>0$ independent of the mesh size.

\subsection{Approximation properties of a Galerkin projection}

We are now ready to introduce a Galerkin-type projection similar to that in Lemma 2.1 of Baker (1976), using the bilinear form

$$
\widetilde{\mathscr{A}}_{h}(\mathbf{u}, \mathbf{v})=\widetilde{a}_{h}(\mathbf{u}, \mathbf{v})+(\mathbf{u}, \mathbf{v})
$$

This form clearly satisfies

$$
\begin{array}{ll}
\widetilde{\mathscr{A}}_{h}(\mathbf{u}, \mathbf{v}) \leqslant \max \left\{1, C_{\text {cont }}\right\}\|\mathbf{u}\|_{h}\|\mathbf{v}\|_{h}, & \mathbf{u}, \mathbf{v} \in \mathbf{V}(h), \\
\widetilde{\mathscr{A}}_{h}(\mathbf{u}, \mathbf{u}) \geqslant \min \left\{1, C_{\text {coer }}\right\}\|\mathbf{u}\|_{h}^{2}, & \mathbf{u} \in \mathbf{V}_{h},
\end{array}
$$

with $C_{\text {coer }}$ and $C_{\text {cont }}$ denoting the constants from (5.1) and (5.2), respectively.

Let now $\mathbf{u} \in H^{\mathrm{s}}(\Omega)^{3}$, with $\nabla \times \mathbf{u} \in H^{\mathrm{s}}(\Omega)^{3}$ for $s>\frac{1}{2}$. We define the projection $\mathbf{w}^{h} \in \mathbf{V}^{h}$ of $\mathbf{u}$ by

$$
\tilde{\mathscr{A}}_{h}\left(\mathbf{w}^{h}, \mathbf{v}\right)=\tilde{\mathscr{A}}_{h}(\mathbf{u}, \mathbf{v})-r_{h}(\mathbf{u} ; \mathbf{v}) \quad \forall \mathbf{v} \in \mathbf{V}^{h}
$$

In view of the approximation property in (5.5) and the stability of $\tilde{\mathscr{A}}_{h}$ in (5.18), the standard LaxMilgram theorem implies that $\mathbf{w}^{h}$ is well defined. We further note the following key property of the error $\mathbf{u}-\mathbf{w}^{h}$, namely, that it is discretely divergence free.

LEMMA 5.1 Let $\mathbf{w}^{h}$ be the projection of $\mathbf{u}$ defined in (5.19). Then, we have

$$
\left(\mathbf{u}-\mathbf{w}^{h}, \nabla \varphi^{h}\right)=0 \quad \forall \varphi^{h} \in S^{h} .
$$

Proof. Let $\varphi^{h} \in S^{h}$. Since $\nabla S^{h} \subset \mathbf{V}^{c} \subset H_{0}$ (curl; $\Omega$ ), we have that $\llbracket \nabla \varphi^{h} \rrbracket_{\mathrm{T}}=\mathbf{0}$ over any face in $\mathscr{F}_{h}$. Furthermore, $\nabla \times \nabla \varphi^{h}=0$. We thus conclude that

$$
\widetilde{a}_{h}\left(\mathbf{u}-\mathbf{w}^{h}, \nabla \varphi^{h}\right)=0 .
$$


This identity, the definition of $\mathbf{w}^{h}$ in (5.19) and the property in (5.4) then yield

$$
\left(\mathbf{u}-\mathbf{w}^{h}, \nabla \varphi^{h}\right)=\widetilde{a}_{h}\left(\mathbf{u}-\mathbf{w}^{h}, \nabla \varphi^{h}\right)+\left(\mathbf{u}-\mathbf{w}^{h}, \nabla \varphi^{h}\right)=\widetilde{\mathscr{A}}_{h}\left(\mathbf{u}-\mathbf{w}^{h}, \nabla \varphi^{h}\right)=r_{h}\left(\mathbf{u} ; \nabla \varphi^{h}\right)=0 .
$$

This proves the assertion.

We now estimate the error $\mathbf{u}-\mathbf{w}^{h}$ in the energy norm.

LEMMA 5.2 Let $\mathbf{w}^{h}$ be the projection of $\mathbf{u}$ defined in (5.19). Then, we have

$$
\left\|\mathbf{u}-\mathbf{w}^{h}\right\|_{h} \leqslant C_{E} h^{\min \{s, \ell\}}\left(\|\mathbf{u}\|_{s}+\|\nabla \times \mathbf{u}\|_{s}\right),
$$

with a constant $C_{E}>0$ that is independent of the mesh size.

Proof. We first use the triangle inequality and obtain

$$
\left\|\mathbf{u}-\mathbf{w}^{h}\right\|_{h} \leqslant\left\|\mathbf{u}-\Pi_{\mathrm{N}} \mathbf{u}\right\|_{h}+\left\|\Pi_{\mathrm{N}} \mathbf{u}-\mathbf{w}^{h}\right\|_{h} .
$$

From the approximation property (5.8), we immediately conclude that

$$
\left\|\mathbf{u}-\Pi_{\mathrm{N}} \mathbf{u}\right\|_{h} \leqslant C_{\mathrm{N}} h^{\min \{s, \ell\}}\left(\|\mathbf{u}\|_{s}+\|\nabla \times \mathbf{u}\|_{s}\right) .
$$

It remains to bound $\left\|\Pi_{\mathrm{N}} \mathbf{u}-\mathbf{w}^{h}\right\|_{h}$. From the stability (5.18) of the form $\widetilde{\mathscr{A}}_{h}$, the definition of $\mathbf{w}^{h}$ and the approximation results in (5.5) and (5.8), we conclude that

$$
\begin{aligned}
\min \left\{1, C_{\text {coer }}\right\}\left\|\Pi_{\mathrm{N}} \mathbf{u}-\mathbf{w}^{h}\right\|_{h}^{2} & \leqslant \widetilde{\mathscr{A}}_{h}\left(\Pi_{\mathrm{N}} \mathbf{u}-\mathbf{w}^{h}, \Pi_{\mathrm{N}} \mathbf{u}-\mathbf{w}^{h}\right) \\
& =\widetilde{\mathscr{A}}_{h}\left(\Pi_{\mathrm{N}} \mathbf{u}-\mathbf{u}, \Pi_{\mathrm{N}} \mathbf{u}-\mathbf{w}^{h}\right)+\widetilde{\mathscr{A}_{h}}\left(\mathbf{u}-\mathbf{w}^{h}, \Pi_{\mathrm{N}} \mathbf{u}-\mathbf{w}^{h}\right) \\
& =\widetilde{\mathscr{A}}_{h}\left(\Pi_{\mathrm{N}} \mathbf{u}-\mathbf{u}, \Pi_{\mathrm{N}} \mathbf{u}-\mathbf{w}^{h}\right)+r_{h}\left(\mathbf{u} ; \Pi_{\mathrm{N}} \mathbf{u}-\mathbf{w}^{h}\right) \\
& \leqslant\left(\max \left\{1, C_{\mathrm{cont}}\right\} C_{\mathrm{N}}+C_{\mathrm{R}}\right) h^{\min \{s, \ell\}}\left(\|\mathbf{u}\|_{s}+\|\nabla \times \mathbf{u}\|_{s}\right)\left\|\Pi_{\mathrm{N}} \mathbf{u}-\mathbf{w}^{h}\right\|_{h} .
\end{aligned}
$$

Thus,

$$
\left\|\Pi_{\mathrm{N}} \mathbf{u}-\mathbf{w}^{h}\right\|_{h} \leqslant C h^{\min \{s, \ell\}}\left(\|\mathbf{u}\|_{s}+\|\nabla \times \mathbf{u}\|_{s}\right) .
$$

This completes the proof.

Next, we state and prove an $L^{2}$-norm estimate for $\left\|\mathbf{u}-\mathbf{w}^{h}\right\|_{0}$, using similar ideas to those developed in Section 6 of Houston et al. (2005).

LeMmA 5.3 Let $\mathbf{u} \in H^{\mathrm{s}+\sigma_{E}}(\Omega)^{3}$ be so that $\nabla \times \mathbf{u} \in H^{\mathrm{s}}(\Omega)^{3}$ for $s>\frac{1}{2}$, and let $\mathbf{w}^{h}$ be the projection of $\mathbf{u}$ defined in (5.19). Then, we have the $L^{2}$-norm error bound

$$
\left\|\mathbf{u}-\mathbf{w}^{h}\right\|_{0} \leqslant C_{\mathrm{L}} h^{\min \{s, \ell\}+\sigma_{E}}\left(\|\mathbf{u}\|_{s+\sigma_{E}}+\|\nabla \times \mathbf{u}\|_{s}\right),
$$

with a constant $C_{\mathrm{L}}>0$ that is independent of the mesh size.

Proof. Let $\mathbf{w}^{c} \in \mathbf{V}^{c}$ be the conforming approximation of $\mathbf{w}^{h}$ from (5.15) to (5.16). We have

$$
\left\|\mathbf{u}-\mathbf{w}^{h}\right\|_{0}^{2}=\left(\mathbf{u}-\mathbf{w}^{h}, \mathbf{u}-\Pi_{\mathrm{N}} \mathbf{u}\right)+\left(\mathbf{u}-\mathbf{w}^{h}, \mathbf{w}^{c}-\mathbf{w}^{h}\right)+\left(\mathbf{u}-\mathbf{w}^{h}, \Pi_{\mathrm{N}} \mathbf{u}-\mathbf{w}^{c}\right) .
$$

By the Cauchy-Schwarz inequality, we obtain

$$
\left\|\mathbf{u}-\mathbf{w}^{h}\right\|_{0} \leqslant\left\|\mathbf{u}-\Pi_{\mathrm{N}} \mathbf{u}\right\|_{0}+\left\|\mathbf{w}^{c}-\mathbf{w}^{h}\right\|_{0}+\frac{\left|\left(\mathbf{u}-\mathbf{w}^{h}, \Pi_{\mathrm{N}} \mathbf{u}-\mathbf{w}^{c}\right)\right|}{\left\|\mathbf{u}-\mathbf{w}^{h}\right\|_{0}} .
$$


From the approximation property in (5.15) and the fact that the tangential jumps of $\mathbf{u}$ vanish, we conclude that

$$
\begin{aligned}
\left\|\mathbf{w}^{c}-\mathbf{w}^{h}\right\|_{0}^{2} & \leqslant C_{A}^{2} \sum_{f \in \mathscr{F}_{h}} \int_{f} \mathrm{~h} \mid \llbracket\left[\left.\mathbf{w}^{h} \rrbracket_{\mathrm{T}}\right|^{2} \mathrm{~d} A\right. \\
& =C_{A}^{2} \sum_{f \in \mathscr{F}_{h}} \int_{f} \mathrm{~h}\left|\llbracket \mathbf{u}-\mathbf{w}^{h} \rrbracket_{\mathrm{T}}\right|^{2} \mathrm{~d} A \leqslant C_{A}^{2} h^{2}\left\|\mathbf{u}-\mathbf{w}^{h}\right\|_{h}^{2} .
\end{aligned}
$$

Therefore,

$$
\left\|\mathbf{u}-\mathbf{w}^{h}\right\|_{0} \leqslant\left\|\mathbf{u}-\Pi_{\mathrm{N}} \mathbf{u}\right\|_{0}+C_{A} h\left\|\mathbf{u}-\mathbf{w}^{h}\right\|_{h}+\frac{\left|\left(\mathbf{u}-\mathbf{w}^{h}, \Pi_{\mathrm{N}} \mathbf{u}-\mathbf{w}^{c}\right)\right|}{\left\|\mathbf{u}-\mathbf{w}^{h}\right\|_{0}} .
$$

We claim that the last term on the right-hand side above can be bounded as follows:

$$
\frac{\left|\left(\mathbf{u}-\mathbf{w}^{h}, \Pi_{\mathrm{N}} \mathbf{u}-\mathbf{w}^{c}\right)\right|}{\left\|\mathbf{u}-\mathbf{w}^{h}\right\|_{0}} \leqslant C\left\|\mathbf{u}-\Pi_{\mathrm{N}} \mathbf{u}\right\|_{0}+C h^{\sigma_{E}}\left(\left\|\mathbf{u}-\Pi_{\mathrm{N}} \mathbf{u}\right\|_{h}+\left\|\mathbf{u}-\mathbf{w}^{h}\right\|_{h}\right) .
$$

Provided that (5.21) holds, the bound for $\left\|\mathbf{u}-\mathbf{w}^{h}\right\|_{0}$ follows by using the approximation results for $\Pi_{\mathrm{N}}$ in (5.8) and (5.9) and the bound for $\left\|\mathbf{u}-\mathbf{w}^{h}\right\|_{h}$ in Lemma 5.2:

$$
\left\|\mathbf{u}-\mathbf{w}^{h}\right\|_{0} \leqslant C h^{\min \left\{s+\sigma_{E}, \ell+1\right\}}\|\mathbf{u}\|_{s+\sigma_{E}}+C h^{\min \{s, \ell\}+\sigma_{E}}\left(\|\mathbf{u}\|_{s}+\|\nabla \times \mathbf{u}\|_{s}\right) .
$$

Here, we have also used that $h^{\sigma_{E}} \geqslant C h$ for $\sigma_{E} \in\left(\frac{1}{2}, 1\right]$. Finally, since we have $\|\mathbf{u}\|_{s} \leqslant\|\mathbf{u}\|_{s+\sigma_{E}}$ and $\min \left\{s+\sigma_{E}, \ell+1\right\} \geqslant \min \{s, \ell\}+\sigma_{E}$, the desired bound follows.

Proof of (5.21). It remains to prove the bound (5.21). To do so, we proceed in several steps.

Step 1: Preliminaries: We start by invoking the discrete Helmholtz decomposition in (5.12) and write

$$
\Pi_{\mathrm{N}} \mathbf{u}-\mathbf{w}^{c}=\mathbf{w}^{0}+\nabla r
$$

with $\mathbf{w}^{0} \in \mathbf{X}^{h}$ and $r \in S^{h}$. Let $\mathbf{w}=\mathbf{H w}^{0} \in H_{0}(\operatorname{curl} ; \Omega)$ be the exactly divergence-free approximation of $\mathbf{w}^{0}$ from (5.13). The orthogonality property of $\mathbf{u}-\mathbf{w}^{h}$ in Lemma 5.1 yields

$$
\left(\mathbf{u}-\mathbf{w}^{h}, \Pi_{\mathrm{N}} \mathbf{u}-\mathbf{w}^{c}\right)=\left(\mathbf{u}-\mathbf{w}^{h}, \mathbf{w}^{0}\right)=\left(\mathbf{u}-\mathbf{w}^{h}, \mathbf{w}^{0}-\mathbf{w}\right)+\left(\mathbf{u}-\mathbf{w}^{h}, \mathbf{w}\right) .
$$

Therefore,

$$
\frac{\left|\left(\mathbf{u}-\mathbf{w}^{h}, \Pi_{\mathrm{N}} \mathbf{u}-\mathbf{w}^{c}\right)\right|}{\left\|\mathbf{u}-\mathbf{w}^{h}\right\|_{0}} \leqslant\left\|\mathbf{w}^{0}-\mathbf{w}\right\|_{0}+\|\mathbf{w}\|_{0},
$$

and it is sufficient to estimate $\left\|\mathbf{w}^{0}-\mathbf{w}\right\|_{0}$ and $\|\mathbf{w}\|_{0}$.

Step 2: Estimate of $\left\|\mathbf{w}^{0}-\mathbf{w}\right\|_{0}$ : We claim that

$$
\left\|\mathbf{w}^{0}-\mathbf{w}\right\|_{0} \leqslant C h^{\sigma_{E}}\left(\left\|\mathbf{u}-\Pi_{\mathrm{N}} \mathbf{u}\right\|_{h}+\left\|\mathbf{u}-\mathbf{w}^{h}\right\|_{h}\right) .
$$

To prove (5.24), we first note that

$$
\nabla \times \mathbf{w}=\nabla \times \mathbf{w}^{0}=\nabla \times\left(\Pi_{\mathrm{N}} \mathbf{u}-\mathbf{w}^{c}\right),
$$


in view of the definition of $\mathbf{H}$ and (5.22). Thus, the approximation property (5.13) of the operator $\mathbf{H}$ and the triangle inequality yield

$$
\begin{aligned}
\left\|\mathbf{w}^{0}-\mathbf{w}\right\|_{0} & \leqslant C_{H} h^{\sigma_{E}}\left\|\nabla \times\left(\Pi_{\mathrm{N}} \mathbf{u}-\mathbf{w}^{c}\right)\right\|_{0} \\
& \leqslant C_{H} h^{\sigma_{E}}\left(\left\|\mathbf{u}-\Pi_{\mathrm{N}} \mathbf{u}\right\|_{h}+\left\|\mathbf{u}-\mathbf{w}^{h}\right\|_{h}+\left\|\mathbf{w}^{h}-\mathbf{w}^{c}\right\|_{h}\right) .
\end{aligned}
$$

By using (5.16) and proceeding similarly to (5.20), we obtain

$$
\begin{aligned}
\left\|\mathbf{w}^{h}-\mathbf{w}^{c}\right\|_{h}^{2} & \leqslant C_{A}^{2} \sum_{f \in \mathscr{F}_{h}} \int_{f} \mathrm{~h}^{-1}\left|\llbracket \mathbf{w}^{h} \rrbracket_{\mathrm{T}}\right|^{2} \mathrm{~d} A \\
& =C_{A}^{2} \sum_{f \in \mathscr{F}_{h}} \int_{f} \mathrm{~h}^{-1}\left|\llbracket \mathbf{u}-\mathbf{w}^{h} \rrbracket_{\mathrm{T}}\right|^{2} \mathrm{~d} A \leqslant C_{A}^{2}\left\|\mathbf{u}-\mathbf{w}^{h}\right\|_{h}^{2} .
\end{aligned}
$$

This yields

$$
\left\|\mathbf{w}^{0}-\mathbf{w}\right\|_{0} \leqslant C h^{\sigma_{E}}\left(\left\|\mathbf{u}-\Pi_{\mathrm{N}} \mathbf{u}\right\|_{h}+\left\|\mathbf{u}-\mathbf{w}^{h}\right\|_{h}\right),
$$

which completes the proof of (5.24).

Step 3. Estimate of $\|\mathbf{w}\|_{0}$ : There holds

$$
\|\mathbf{w}\|_{0} \leqslant C\left\|\mathbf{u}-\Pi_{\mathrm{N}} \mathbf{u}\right\|_{0}+C h^{\sigma_{E}}\left(\left\|\mathbf{u}-\Pi_{\mathrm{N}} \mathbf{u}\right\|_{h}+\left\|\mathbf{u}-\mathbf{w}^{h}\right\|_{h}\right)
$$

We will prove this bound by using a duality approach as in Section 6.1 of Houston et al. (2005). To this end, let $\mathbf{z}$ be the solution of the dual problem

$$
\begin{array}{rlr}
\nabla \times(\nabla \times \mathbf{z})+\mathbf{z}=\mathbf{w} & & \text { in } \Omega, \\
\mathbf{n} \times \mathbf{z}=\mathbf{0} & & \text { on } \Gamma .
\end{array}
$$

We conclude from (4.2) that $\mathbf{z}$ and $\nabla \times \mathbf{z}$ belong to $H^{\sigma_{E}}(\Omega)^{3}$ and satisfy

$$
\|\mathbf{z}\|_{\sigma_{E}}+\|\nabla \times \mathbf{z}\|_{\sigma_{E}} \leqslant C_{\mathrm{S}}\|\mathbf{w}\|_{0}
$$

For later use, we note that we have

$$
\widetilde{\mathscr{A}}_{h}(\mathbf{z}, \mathbf{v})-(\mathbf{w}, \mathbf{v})=r_{h}(\mathbf{z} ; \mathbf{v})
$$

for all $\mathbf{v} \in \mathbf{V}^{h}$. In the sequel, it is convenient to also introduce the conforming bilinear form

$$
\mathscr{A}(\mathbf{u}, \mathbf{v})=(\nabla \times \mathbf{u}, \nabla \times \mathbf{v})+(\mathbf{u}, \mathbf{v}), \quad \mathbf{u}, \mathbf{v} \in H_{0}(\operatorname{curl} ; \Omega) .
$$

Obviously, we have $\mathscr{A}(\mathbf{u}, \mathbf{v})=\widetilde{A}_{h}(\mathbf{u}, \mathbf{v})$ for all $\mathbf{u}, \mathbf{v} \in H_{0}(\operatorname{curl} ; \Omega)$. Multiplying the dual problem with $\mathbf{w}$ and integrating by parts, we obtain

$$
\|\mathbf{w}\|_{0}^{2}=\mathscr{A}(\mathbf{z}, \mathbf{w})=\mathscr{A}\left(\mathbf{z}-\Pi^{c} \mathbf{z}, \mathbf{w}\right)+\mathscr{A}\left(\Pi^{c} \mathbf{z}, \mathbf{w}\right),
$$

where $\Pi^{c}$ is the projection from (5.10). 
Since $\nabla \times \mathbf{w}=\nabla \times \mathbf{w}^{0}$ and by the definition of $\Pi^{c}$, we infer that

$$
\begin{aligned}
\mathscr{A}\left(\mathbf{z}-\Pi^{c} \mathbf{z}, \mathbf{w}\right) & =\left(\nabla \times\left(\mathbf{z}-\Pi^{c} \mathbf{z}\right), \nabla \times \mathbf{w}^{0}\right)+\left(\mathbf{z}-\Pi^{c} \mathbf{z}, \mathbf{w}\right) \\
& =-\left(\mathbf{z}-\Pi^{c} \mathbf{z}, \mathbf{w}^{0}\right)+\left(\mathbf{z}-\Pi^{c} \mathbf{z}, \mathbf{w}\right)=\left(\mathbf{z}-\Pi^{c} \mathbf{z}, \mathbf{w}-\mathbf{w}^{0}\right) .
\end{aligned}
$$

The approximation result for $\Pi^{c}$ in (5.11) and the bound in (5.27) yield

$$
\left\|\mathbf{z}-\Pi^{c} \mathbf{z}\right\|_{0} \leqslant\left\|\mathbf{z}-\Pi^{c} \mathbf{z}\right\|_{h} \leqslant C_{\mathrm{N}} h^{\sigma_{E}}\left(\|\mathbf{z}\|_{\sigma_{E}}+\|\nabla \times \mathbf{z}\|_{\sigma_{\mathrm{E}}}\right) \leqslant C_{\mathrm{N}} C_{\mathrm{S}} h^{\sigma_{E}}\|\mathbf{w}\|_{0} .
$$

For later use, we also point out that the dual bound (5.27) implies

$$
\begin{aligned}
\left\|\Pi^{c} \mathbf{z}\right\|_{0} & \leqslant\left\|\mathbf{z}-\Pi^{c} \mathbf{z}\right\|_{0}+\|\mathbf{z}\|_{0} \\
& \leqslant C_{\mathrm{N}} h^{\sigma_{E}}\left(\|\mathbf{z}\|_{\sigma_{E}}+\|\nabla \times \mathbf{z}\|_{\sigma_{E}}\right)+\|\mathbf{z}\|_{\sigma_{E}} \leqslant C\|\mathbf{w}\|_{0} .
\end{aligned}
$$

The Cauchy-Schwarz inequality and the estimates in (5.24) and (5.31) thus yield

$$
\begin{aligned}
\left|\mathscr{A}\left(\mathbf{z}-\Pi^{c} \mathbf{z}, \mathbf{w}\right)\right| & \leqslant\left\|\mathbf{z}-\Pi^{c} \mathbf{z}\right\|_{0}\left\|\mathbf{w}-\mathbf{w}^{0}\right\|_{0} \\
& \leqslant C h^{2 \sigma_{E}}\|\mathbf{w}\|_{0}\left(\left\|\mathbf{u}-\Pi_{\mathrm{N}} \mathbf{u}\right\|_{h}+\left\|\mathbf{u}-\mathbf{w}^{h}\right\|_{h}\right) .
\end{aligned}
$$

Finally, we need to bound the term $\mathscr{A}\left(\Pi^{c} \mathbf{z}, \mathbf{w}\right)$ in (5.29). To this end, in view of (5.10), (5.22) and since $\nabla \times \mathbf{w}=\nabla \times \mathbf{w}^{0}$, we conclude that

$$
\begin{aligned}
\mathscr{A}\left(\Pi^{c} \mathbf{z}, \mathbf{w}\right) & =\left(\nabla \times \Pi^{c} \mathbf{z}, \nabla \times \mathbf{w}\right)+\left(\Pi^{c} \mathbf{z}, \mathbf{w}\right) \\
& =\left(\nabla \times \Pi^{c} \mathbf{z}, \nabla \times\left(\Pi_{\mathrm{N}} \mathbf{u}-\mathbf{w}^{c}\right)\right)+\left(\Pi^{c} \mathbf{z}, \mathbf{w}-\mathbf{w}^{0}\right)+\left(\Pi^{c} \mathbf{z}, \mathbf{w}^{0}\right) \\
& =\left(\nabla \times \Pi^{c} \mathbf{z}, \nabla \times\left(\Pi_{\mathrm{N}} \mathbf{u}-\mathbf{w}^{c}\right)\right)+\left(\Pi^{c} \mathbf{z}, \mathbf{w}-\mathbf{w}^{0}\right)+\left(\Pi^{c} \mathbf{z}, \Pi_{\mathrm{N}} \mathbf{u}-\mathbf{w}^{c}\right) \\
& =\mathscr{A}\left(\Pi^{c} \mathbf{z}, \Pi_{\mathrm{N}} \mathbf{u}-\mathbf{w}^{c}\right)+\left(\Pi^{c} \mathbf{z}, \mathbf{w}-\mathbf{w}^{0}\right) \\
& =\mathscr{A}\left(\mathbf{z}, \Pi_{\mathrm{N}} \mathbf{u}-\mathbf{w}^{c}\right)+\left(\Pi^{c} \mathbf{z}, \mathbf{w}-\mathbf{w}^{0}\right) .
\end{aligned}
$$

Here, we have also used that

$$
\left(\Pi^{c} \mathbf{z}, \nabla r\right)=(\mathbf{z}, \nabla r)=0,
$$

which follows readily from the definition of $\Pi^{c}$ and the fact that $\mathbf{z}$ is divergence free. Since we have

$$
\mathscr{A}\left(\mathbf{z}, \Pi_{\mathrm{N}} \mathbf{u}-\mathbf{w}^{c}\right)=\mathscr{A}\left(\mathbf{z}, \Pi_{\mathrm{N}} \mathbf{u}-\mathbf{u}\right)+\widetilde{\mathscr{A}}_{h}\left(\mathbf{z}, \mathbf{u}-\mathbf{w}^{h}\right)+\widetilde{\mathscr{A}}_{h}\left(\mathbf{z}, \mathbf{w}^{h}-\mathbf{w}^{c}\right)
$$

we obtain

$$
\mathscr{A}\left(\Pi^{c} \mathbf{z}, \mathbf{w}\right)=\left(\Pi^{c} \mathbf{z}, \mathbf{w}-\mathbf{w}^{0}\right)+\mathscr{A}\left(\mathbf{z}, \Pi_{\mathrm{N}} \mathbf{u}-\mathbf{u}\right)+\widetilde{\mathscr{A}}_{h}\left(\mathbf{z}, \mathbf{u}-\mathbf{w}^{h}\right)+\widetilde{\mathscr{A}}_{h}\left(\mathbf{z}, \mathbf{w}^{h}-\mathbf{w}^{c}\right) .
$$

We now bound the four terms in (5.34). For the first term, we use the stability estimate for $\left\|\Pi^{c} \mathbf{z}\right\|_{0}$ in (5.32) and the bound for $\left\|\mathbf{w}-\mathbf{w}^{0}\right\|_{0}$ in (5.24):

$$
\begin{aligned}
\left|\left(\Pi^{c} \mathbf{z}, \mathbf{w}-\mathbf{w}^{0}\right)\right| & \leqslant\left\|\Pi^{c} \mathbf{z}\right\|_{0}\left\|\mathbf{w}-\mathbf{w}^{0}\right\|_{0} \\
& \leqslant C\|\mathbf{w}\|_{0}\left\|\mathbf{w}-\mathbf{w}^{0}\right\|_{0} \\
& \leqslant C h^{\sigma_{E}}\|\mathbf{w}\|_{0}\left(\left\|\mathbf{u}-\Pi_{\mathrm{N}} \mathbf{u}\right\|_{h}+\left\|\mathbf{u}-\mathbf{w}^{h}\right\|_{h}\right) .
\end{aligned}
$$


From the definition of the dual problem and the Cauchy-Schwarz inequality, we conclude that the second term in (5.34) can be estimated as follows:

$$
\left|\mathscr{A}\left(\mathbf{z}, \Pi_{\mathrm{N}} \mathbf{u}-\mathbf{u}\right)\right|=\left|\left(\mathbf{w}, \Pi_{\mathrm{N}} \mathbf{u}-\mathbf{u}\right)\right| \leqslant\|\mathbf{w}\|_{0}\left\|\mathbf{u}-\Pi_{\mathrm{N}} \mathbf{u}\right\|_{0} \leqslant\|\mathbf{w}\|_{0}\left\|\mathbf{u}-\Pi_{\mathrm{N}} \mathbf{u}\right\|_{0} .
$$

To bound the third term in (5.34), we first employ the symmetry of $\widetilde{\mathscr{A}_{h}}(\cdot, \cdot)$, the definition of $\mathbf{w}^{h}$ in (5.19) and identity (5.4):

$$
\widetilde{\mathscr{A}}_{h}\left(\mathbf{z}, \mathbf{u}-\mathbf{w}^{h}\right)=\widetilde{\mathscr{A}}_{h}\left(\mathbf{u}-\mathbf{w}^{h}, \mathbf{z}\right)=\widetilde{\mathscr{A}}_{h}\left(\mathbf{u}-\mathbf{w}^{h}, \mathbf{z}-\Pi_{\mathrm{N}} \mathbf{z}\right) .
$$

The continuity of $\widetilde{\mathscr{A}}_{h}$, the approximation property of $\Pi_{\mathrm{N}}$ in (5.8) and the stability (5.27) of the dual problem thus give

$$
\begin{aligned}
\left|\widetilde{\mathscr{A}_{h}}\left(\mathbf{z}, \mathbf{u}-\mathbf{w}^{h}\right)\right| & \leqslant C\left\|\mathbf{u}-\mathbf{w}^{h}\right\|_{h}\left\|\mathbf{z}-\Pi_{\mathrm{N}} \mathbf{z}\right\|_{h} \\
& \leqslant C h^{\sigma_{E}}\left\|\mathbf{u}-\mathbf{w}^{h}\right\|_{h}\left(\|\mathbf{z}\|_{\sigma_{E}}+\|\nabla \times \mathbf{z}\|_{\sigma_{\mathrm{E}}}\right) \\
& \leqslant C h^{\sigma_{E}}\|\mathbf{w}\|_{0}\left\|\mathbf{u}-\mathbf{w}^{h}\right\|_{h} .
\end{aligned}
$$

The variational problem in (5.28) implies that the fourth term in (5.34) can be written as

$$
\widetilde{\mathscr{A}}_{h}\left(\mathbf{z}, \mathbf{w}^{h}-\mathbf{w}^{c}\right)=\left(\mathbf{w}, \mathbf{w}^{h}-\mathbf{w}^{c}\right)+r_{h}\left(\mathbf{z} ; \mathbf{w}^{h}-\mathbf{w}^{c}\right) .
$$

Then, from (5.5) and the stability of the dual problem in (5.27), we have

$$
\begin{aligned}
\left|\tilde{\mathscr{A}}_{h}\left(\mathbf{z}, \mathbf{w}^{h}-\mathbf{w}^{c}\right)\right| & \leqslant\|\mathbf{w}\|_{0}\left\|\mathbf{w}^{h}-\mathbf{w}^{c}\right\|_{0}+C_{\mathrm{R}} h^{\sigma_{E}}\left\|\mathbf{w}^{h}-\mathbf{w}^{c}\right\|_{h}\|\nabla \times \mathbf{z}\|_{\sigma_{E}} \\
& \leqslant\|\mathbf{w}\|_{0}\left\|\mathbf{w}^{h}-\mathbf{w}^{c}\right\|_{0}+C_{\mathrm{R}} C_{\mathrm{S}} h^{\sigma_{E}}\|\mathbf{w}\|_{0}\left\|\mathbf{w}^{h}-\mathbf{w}^{c}\right\|_{h} .
\end{aligned}
$$

Recall from (5.20) and (5.25) that

$$
\begin{aligned}
& \left\|\mathbf{w}^{h}-\mathbf{w}^{c}\right\|_{0} \leqslant C_{A} h\left\|\mathbf{u}-\mathbf{w}^{h}\right\|_{h}, \\
& \left\|\mathbf{w}^{h}-\mathbf{w}^{c}\right\|_{h} \leqslant C_{A}\left\|\mathbf{u}-\mathbf{w}^{h}\right\|_{h} .
\end{aligned}
$$

Hence,

$$
\left|\widetilde{\mathscr{A}_{h}}\left(\mathbf{z}, \mathbf{w}^{h}-\mathbf{w}^{c}\right)\right| \leqslant C h^{\sigma_{E}}\|\mathbf{w}\|_{0}\left\|\mathbf{u}-\mathbf{w}^{h}\right\|_{h} .
$$

The equality in (5.34) and the above four bounds yield

$$
\mathscr{A}\left(\Pi^{c} \mathbf{z}, \mathbf{w}\right) \leqslant\|\mathbf{w}\|_{0}\left\|\mathbf{u}-\Pi_{\mathrm{N}} \mathbf{u}\right\|_{0}+C h^{\sigma_{E}}\|\mathbf{w}\|_{0}\left(\left\|\mathbf{u}-\Pi_{\mathrm{N}} \mathbf{u}\right\|_{h}+\left\|\mathbf{u}-\mathbf{w}^{h}\right\|_{h}\right) .
$$

The bound in (5.26) then follows by combining (5.29), (5.33) and (5.35).

Step 4. The claim in (5.21) now follows from (5.23), (5.24) and (5.26).

\subsection{Proof of Theorem 4.3}

Let now the solution $\mathbf{u}$ of the Maxwell's equations (2.1) satisfy the regularity assumption in Theorem 4.3. We define $\mathbf{w}^{h}(t)=\mathbf{w}^{h}(\cdot, t) \in \mathbf{V}^{h}$ by

$$
\widetilde{\mathscr{A}}_{h}\left(\mathbf{w}^{h}(t), \mathbf{v}\right)=\widetilde{\mathscr{A}}_{h}(\mathbf{u}(t), \mathbf{v})-r_{h}(\mathbf{u}(t) ; \mathbf{v}), \quad \mathbf{v} \in \mathbf{V}^{h} \text {, a.e. in } J .
$$


It can be readily seen that $\mathbf{w}^{h} \in L^{\infty}\left(J ; \mathbf{V}^{h}\right)$. Moreover, we have $\mathbf{w}_{t}^{h} \in L^{\infty}\left(J ; \mathbf{V}^{h}\right)$ and

$$
\tilde{\mathscr{A}}_{h}\left(\mathbf{w}_{t}^{h}, \mathbf{v}\right)=\widetilde{\mathscr{A}}_{h}\left(\mathbf{u}_{t}, \mathbf{v}\right)-r_{h}\left(\mathbf{u}_{t} ; \mathbf{v}\right), \quad \mathbf{v} \in \mathbf{V}^{h} \text {, a.e. in } J .
$$

Similarly,

$$
\widetilde{\mathscr{A}}_{h}\left(\mathbf{w}^{h}(0), \mathbf{v}\right)=\widetilde{\mathscr{A}}_{h}\left(\mathbf{u}_{0}, \mathbf{v}\right)-r_{h}\left(\mathbf{u}_{0} ; \mathbf{v}\right), \quad \mathbf{v} \in \mathbf{V}^{h} .
$$

Therefore, Lemma 5.3 immediately implies the following estimates.

LEMMA 5.4 Let $\mathbf{w}^{h}$ be defined by (5.36). Under the regularity assumptions of Theorem 4.3, we have

$$
\begin{aligned}
\left\|\mathbf{u}-\mathbf{w}^{h}\right\|_{L^{\infty}\left(J ; L^{2}(\Omega)^{3}\right)} & \leqslant C_{\mathrm{L}} h^{\min \{s, \ell\}+\sigma_{E}}\left(\|\mathbf{u}\|_{L^{\infty}\left(J ; H^{s+\sigma} E(\Omega)^{3}\right)}+\|\nabla \times \mathbf{u}\|_{L^{\infty}\left(J ; H^{\mathrm{s}}(\Omega)^{3}\right)}\right), \\
\left\|\left(\mathbf{u}-\mathbf{w}^{h}\right)_{t}\right\|_{L^{\infty}\left(J ; L^{2}(\Omega)^{3}\right)} & \leqslant C_{\mathrm{L}} h^{\min \{s, \ell\}+\sigma_{E}}\left(\left\|\mathbf{u}_{t}\right\|_{L^{\infty}\left(J ; H^{\mathrm{s}+\sigma_{E}}(\Omega)^{3}\right)}+\left\|\nabla \times \mathbf{u}_{t}\right\|_{L^{\infty}\left(J ; H^{\mathrm{s}}(\Omega)^{3}\right)}\right), \\
\left\|\left(\mathbf{u}-\mathbf{w}^{h}\right)(0)\right\|_{0} & \leqslant C_{\mathrm{L}} h^{\min \{s, \ell\}+\sigma_{E}}\left(\left\|\mathbf{u}_{0}\right\|_{s+\sigma_{E}}+\left\|\nabla \times \mathbf{u}_{0}\right\|_{s}\right) .
\end{aligned}
$$

The constant $C_{\mathrm{L}}>0$ is as in Lemma 5.3 and $\sigma_{E} \in\left(\frac{1}{2}, 1\right]$ is the stability parameter from (4.2).

We consider the error $\mathbf{u}-\mathbf{u}_{h}$ and use the triangle inequality to write

$$
\|\mathbf{e}\|_{L^{\infty}\left(J ; L^{2}(\Omega)^{3}\right)}^{2} \leqslant 2\left\|\mathbf{u}-\mathbf{w}^{h}\right\|_{L^{\infty}\left(J ; L^{2}(\Omega)^{3}\right)}^{2}+2\left\|\mathbf{u}^{h}-\mathbf{w}^{h}\right\|_{L^{\infty}\left(J ; L^{2}(\Omega)^{3}\right)}^{2} .
$$

The first term can be estimated from the $L^{2}$-bounds in Lemma 5.4. To derive an estimate for the second term, we proceed as follows. First, we fix $\mathbf{v} \in L^{\infty}\left(J ; \mathbf{V}^{h}\right)$ and assume that $\mathbf{v}_{t} \in L^{\infty}\left(J ; \mathbf{V}^{h}\right)$. From the definition of $\mathbf{w}^{h}$, we have

$$
\widetilde{a}_{h}\left(\mathbf{w}^{h}, \mathbf{v}\right)=\widetilde{a}_{h}(\mathbf{u}, \mathbf{v})+\left(\mathbf{u}-\mathbf{w}^{h}, \mathbf{v}\right)-r_{h}(\mathbf{u} ; \mathbf{v}) .
$$

Moreover, the error equation in (5.6) yields

$$
r_{h}(\mathbf{u}, \mathbf{v})-\widetilde{a}_{h}\left(\mathbf{u}-\mathbf{u}^{h}\right)=\left(\sigma \mathbf{e}_{t}, \mathbf{v}\right)+\left(\left(\mathbf{u}-\mathbf{u}^{h}\right)_{t t}, \mathbf{v}\right) .
$$

Therefore, we conclude that

$$
\begin{aligned}
\left(\left(\mathbf{u}^{h}-\mathbf{w}^{h}\right)_{t t}, \mathbf{v}\right)+\widetilde{a}_{h}\left(\mathbf{u}^{h}-\mathbf{w}^{h}, \mathbf{v}\right) & =\left(\mathbf{u}_{t t}^{h}, \mathbf{v}\right)-\widetilde{a}_{h}\left(\mathbf{u}-\mathbf{u}^{h}, \mathbf{v}\right)+r_{h}(\mathbf{u} ; \mathbf{v})-\left(\mathbf{u}-\mathbf{w}^{h}, \mathbf{v}\right)-\left(\mathbf{w}_{t t}^{h}, \mathbf{v}\right) \\
& =\left(\mathbf{u}_{t t}, \mathbf{v}\right)+\left(\sigma \mathbf{e}_{t}, \mathbf{v}\right)-\left(\mathbf{u}-\mathbf{w}^{h}, \mathbf{v}\right)-\left(\mathbf{w}_{t t}^{h}, \mathbf{v}\right) .
\end{aligned}
$$

We rewrite this identity as

$$
\begin{aligned}
& \frac{\mathrm{d}}{\mathrm{d} t}\left(\left(\mathbf{u}^{h}-\mathbf{w}^{h}\right)_{t}, \mathbf{v}\right)-\left(\left(\mathbf{u}^{h}-\mathbf{w}^{h}\right)_{t}, \mathbf{v}_{t}\right)+\widetilde{a}_{h}\left(\mathbf{u}^{h}-\mathbf{w}^{h}, \mathbf{v}\right) \\
& \quad=\frac{\mathrm{d}}{\mathrm{d} t}\left(\left(\mathbf{u}-\mathbf{w}^{h}\right)_{t}, \mathbf{v}\right)-\left(\left(\mathbf{u}-\mathbf{w}^{h}\right)_{t}, \mathbf{v}_{t}\right)+\frac{\mathrm{d}}{\mathrm{d} t}(\sigma \mathbf{e}, \mathbf{v})-\left(\sigma \mathbf{e}, \mathbf{v}_{t}\right)-\left(\mathbf{u}-\mathbf{w}^{h}, \mathbf{v}\right),
\end{aligned}
$$

which yields

$$
\begin{aligned}
-\left(\left(\mathbf{u}^{h}-\mathbf{w}^{h}\right)_{t}, \mathbf{v}_{t}\right)+\widetilde{a}_{h}\left(\mathbf{u}^{h}-\mathbf{w}^{h}, \mathbf{v}\right)= & \frac{\mathrm{d}}{\mathrm{d} t}\left(\mathbf{e}_{t}, \mathbf{v}\right)-\left(\left(\mathbf{u}-\mathbf{w}^{h}\right)_{t}, \mathbf{v}_{t}\right) \\
& +\frac{\mathrm{d}}{\mathrm{d} t}(\sigma \mathbf{e}, \mathbf{v})-\left(\sigma \mathbf{e}, \mathbf{v}_{t}\right)-\left(\mathbf{u}-\mathbf{w}^{h}, \mathbf{v}\right) .
\end{aligned}
$$


Let now $\tau \in[0, T]$ be fixed, and consider the particular function $\widehat{\mathbf{v}}$ given by

$$
\widehat{\mathbf{v}}(t)=\int_{t}^{\tau}\left(\mathbf{u}^{h}-\mathbf{w}^{h}\right)(s) \mathrm{d} s, \quad t \in \bar{J} .
$$

From the definition of $\widehat{\mathbf{v}}(t)$, we infer that

$$
\widehat{\mathbf{v}}(\tau)=\mathbf{0}, \quad \widehat{\mathbf{v}}_{t}(t)=-\left(\mathbf{u}^{h}-\mathbf{w}^{h}\right)(t) .
$$

Thus,

$$
\left\|\widehat{\mathbf{v}}_{t}\right\|_{L^{\infty}\left(J ; L^{2}(\Omega)^{3}\right)} \leqslant\left\|\mathbf{u}^{h}-\mathbf{w}^{h}\right\|_{L^{\infty}\left(J ; L^{2}(\Omega)^{3}\right)} .
$$

Moreover,

$$
\|\widehat{\mathbf{v}}(t)\|_{0} \leqslant \int_{0}^{T}\left\|\left(\mathbf{u}^{h}-\mathbf{w}^{h}\right)(s)\right\|_{0} \mathrm{~d} s \leqslant T\left\|\mathbf{u}^{h}-\mathbf{w}^{h}\right\|_{L^{\infty}\left(J ; L^{2}(\Omega)^{3}\right)} .
$$

Because this bound is independent of $t$, it also holds for the supremum over $t \in J$, i.e.

$$
\|\widehat{\mathbf{v}}\|_{L^{\infty}\left(J ; L^{2}(\Omega)^{3}\right)} \leqslant T\left\|\mathbf{u}^{h}-\mathbf{w}^{h}\right\|_{L^{\infty}\left(J ; L^{2}(\Omega)^{3}\right)} .
$$

Then, we choose $\mathbf{v}=\widehat{\mathbf{v}}$ in (5.38), which yields

$$
\begin{aligned}
\left(\left(\mathbf{u}^{h}-\mathbf{w}^{h}\right)_{t}, \mathbf{u}^{h}-\mathbf{w}^{h}\right)-\widetilde{a}_{h}\left(\widehat{\mathbf{v}}_{t}, \widehat{\mathbf{v}}\right)= & \frac{\mathrm{d}}{\mathrm{d} t}\left(\mathbf{e}_{t}, \widehat{\mathbf{v}}\right)+\left(\left(\mathbf{u}-\mathbf{w}^{h}\right)_{t}, \mathbf{u}^{h}-\mathbf{w}^{h}\right) \\
& +\frac{\mathrm{d}}{\mathrm{d} t}(\sigma \mathbf{e}, \widehat{\mathbf{v}})+\left(\sigma \mathbf{e}, \mathbf{u}^{h}-\mathbf{w}^{h}\right)-\left(\mathbf{u}-\mathbf{w}^{h}, \widehat{\mathbf{v}}\right) .
\end{aligned}
$$

Since the DG form $\widetilde{a}_{h}(\cdot, \cdot)$ is symmetric, we obtain

$$
\begin{aligned}
\frac{1}{2} \frac{\mathrm{d}}{\mathrm{d} t}\left\|\mathbf{u}^{h}-\mathbf{w}^{h}\right\|_{0}^{2}-\frac{1}{2} \frac{\mathrm{d}}{\mathrm{d} t} \widetilde{a}_{h}(\widehat{\mathbf{v}}, \widehat{\mathbf{v}})= & \frac{\mathrm{d}}{\mathrm{d} t}\left(\mathbf{e}_{t}, \widehat{\mathbf{v}}\right)+\left(\left(\mathbf{u}-\mathbf{w}^{h}\right)_{t}, \mathbf{u}^{h}-\mathbf{w}^{h}\right) \\
& +\frac{\mathrm{d}}{\mathrm{d} t}(\sigma \mathbf{e}, \widehat{\mathbf{v}})+\left(\sigma \mathbf{e}, \mathbf{u}^{h}-\mathbf{w}^{h}\right)-\left(\mathbf{u}-\mathbf{w}^{h}, \widehat{\mathbf{v}}\right) .
\end{aligned}
$$

Integration over $[0, \tau]$ and using that $\widehat{\mathbf{v}}(\tau)=\mathbf{0}$ yield

$$
\begin{aligned}
&\left\|\left(\mathbf{u}^{h}-\mathbf{w}^{h}\right)(\tau)\right\|_{0}^{2}-\left\|\left(\mathbf{u}^{h}-\mathbf{w}^{h}\right)(0)\right\|_{0}^{2}+\widetilde{a}_{h}(\widehat{\mathbf{v}}(0), \widehat{\mathbf{v}}(0)) \\
&=-2\left(\mathbf{e}_{t}(0), \widehat{\mathbf{v}}(0)\right)+2 \int_{0}^{\tau}\left(\left(\mathbf{u}-\mathbf{w}^{h}\right)_{t}, \mathbf{u}^{h}-\mathbf{w}^{h}\right) \mathrm{d} t \\
&-2(\sigma \mathbf{e}(0), \widehat{\mathbf{v}}(0))+2 \int_{0}^{\tau}\left(\sigma \mathbf{e}, \mathbf{u}^{h}-\mathbf{w}^{h}\right) \mathrm{d} t-2 \int_{0}^{\tau}\left(\mathbf{u}-\mathbf{w}^{h}, \widehat{\mathbf{v}}\right) \mathrm{d} t \\
&= T_{1}+T_{2}+T_{3}+T_{4}+T_{5} .
\end{aligned}
$$

Since $\mathbf{e}_{t}(0)=\mathbf{v}_{0}-\Pi_{h} \mathbf{v}_{0}$ and $\widehat{\mathbf{v}}(0)$ belongs to $\mathbf{V}^{h}$, we conclude that $T_{1}=0$. Moreover, the positive semidefiniteness of the form $\widetilde{a}_{h}$ ensures that $\widetilde{a}_{h}(\widehat{\mathbf{v}}(0), \widehat{\mathbf{v}}(0)) \geqslant 0$. This leads to the inequality

$$
\left\|\left(\mathbf{u}^{h}-\mathbf{w}^{h}\right)(\tau)\right\|_{0}^{2} \leqslant\left\|\left(\mathbf{u}^{h}-\mathbf{w}^{h}\right)(0)\right\|_{0}^{2}+T_{2}+T_{3}+T_{4}+T_{5} .
$$


The Cauchy-Schwarz inequality, the Hölder's inequality and the geometric-arithmetic mean inequality yield

$$
\begin{aligned}
T_{2} & \leqslant 2 T\left\|\left(\mathbf{u}-\mathbf{w}^{h}\right)_{t}\right\|_{L^{\infty}\left(J ; L^{2}(\Omega)^{3}\right)}\left\|\mathbf{u}^{h}-\mathbf{w}^{h}\right\|_{L^{\infty}\left(J ; L^{2}(\Omega)^{3}\right)} \\
& \leqslant \frac{1}{8}\left\|\mathbf{u}^{h}-\mathbf{w}^{h}\right\|_{L^{\infty}\left(J ; L^{2}(\Omega)^{3}\right)}^{2}+C_{2}\left\|\left(\mathbf{u}-\mathbf{w}^{h}\right)_{t}\right\|_{L^{\infty}\left(J ; L^{2}(\Omega)^{3}\right)^{2}}^{2}
\end{aligned}
$$

Employing the Cauchy-Schwarz inequality, the estimate (5.40) and the geometric-arithmetic mean inequality, we obtain

$$
\begin{aligned}
T_{3} & \leqslant 2 \sigma^{\star}\|\mathbf{e}(0)\|_{0}\|\widehat{\mathbf{v}}(0)\|_{0} \\
& \leqslant 2 \sigma^{\star} T\|\mathbf{e}(0)\|_{0}\left\|\mathbf{u}^{h}-\mathbf{w}^{h}\right\|_{L^{\infty}\left(J ; L^{2}(\Omega)^{3}\right)} \\
& \leqslant \frac{1}{8}\left\|\mathbf{u}^{h}-\mathbf{w}^{h}\right\|_{\left.L^{\infty}(J ; \Omega)^{3}\right)}^{2}+C_{3}\|\mathbf{e}(0)\|_{0}^{2} .
\end{aligned}
$$

Similarly,

$$
\begin{aligned}
T_{4} & =2 \int_{0}^{\tau}\left(\sigma\left(\mathbf{u}-\mathbf{w}^{h}\right), \mathbf{u}^{h}-\mathbf{w}^{h}\right) \mathrm{d} t-2 \int_{0}^{\tau}\left(\sigma\left(\mathbf{u}^{h}-\mathbf{w}^{h}\right), \mathbf{u}^{h}-\mathbf{w}^{h}\right) \mathrm{d} t \\
& \leqslant 2 \sigma^{\star} T\left\|\mathbf{u}-\mathbf{w}^{h}\right\|_{L^{\infty}\left(J ; L^{2}(\Omega)^{3}\right)}\left\|\mathbf{u}^{h}-\mathbf{w}^{h}\right\|_{L^{\infty}\left(J ; L^{2}(\Omega)^{3}\right)} \\
& \leqslant \frac{1}{8}\left\|\mathbf{u}^{h}-\mathbf{w}^{h}\right\|_{L^{\infty}\left(J ; L^{2}(\Omega)^{3}\right)}^{2}+C_{4}\left\|\mathbf{u}-\mathbf{w}^{h}\right\|_{L^{\infty}\left(J ; L^{2}(\Omega)^{3}\right)}^{2},
\end{aligned}
$$

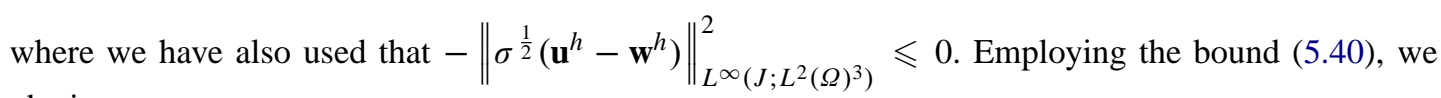
obtain

$$
\begin{aligned}
T_{5} & \leqslant 2 T\left\|\mathbf{u}-\mathbf{w}^{h}\right\|_{L^{\infty}\left(J ; L^{2}(\Omega)^{3}\right)}\|\widehat{\mathbf{v}}\|_{L^{\infty}\left(J ; L^{2}(\Omega)^{3}\right)} \\
& \leqslant 2 T^{2}\left\|\mathbf{u}-\mathbf{w}^{h}\right\|_{L^{\infty}\left(J ; L^{2}(\Omega)^{3}\right)}\left\|\mathbf{u}^{h}-\mathbf{w}^{h}\right\|_{L^{\infty}\left(J ; L^{2}(\Omega)^{3}\right)} \\
& \leqslant \frac{1}{8}\left\|\mathbf{u}^{h}-\mathbf{w}^{h}\right\|_{L^{\infty}\left(J ; L^{2}(\Omega)^{3}\right)}^{2}+C_{5}\left\|\mathbf{u}-\mathbf{w}^{h}\right\|_{L^{\infty}\left(J ; L^{2}(\Omega)^{3}\right)}^{2} .
\end{aligned}
$$

The upper bounds for $T_{2}, T_{3}, T_{4}$ and $T_{5}$ are independent of $\tau$. Taking the supremum over $\tau \in J$ in the inequality (5.42), we thus obtain the estimate

$$
\begin{aligned}
\left\|\mathbf{u}^{h}-\mathbf{w}^{h}\right\|_{L^{\infty}\left(J ; L^{2}(\Omega)^{3}\right)}^{2} \leqslant & \frac{1}{2}\left\|\mathbf{u}^{h}-\mathbf{w}^{h}\right\|_{L^{\infty}\left(J ; L^{2}(\Omega)^{3}\right)}^{2}+\left\|\left(\mathbf{u}^{h}-\mathbf{w}^{h}\right)(0)\right\|_{0}^{2}+C\|\mathbf{e}(0)\|_{0}^{2} \\
& +C\left\|\left(\mathbf{u}-\mathbf{w}^{h}\right)_{t}\right\|_{L^{\infty}\left(J ; L^{2}(\Omega)^{3}\right)}^{2}+C\left\|\mathbf{u}-\mathbf{w}^{h}\right\|_{L^{\infty}\left(J ; L^{2}(\Omega)^{3}\right)^{3}}^{2}
\end{aligned}
$$

This leads to

$$
\begin{aligned}
\left\|\mathbf{u}^{h}-\mathbf{w}^{h}\right\|_{L^{\infty}\left(J ; L^{2}(\Omega)^{3}\right)}^{2} \leqslant & C\left\|\left(\mathbf{u}-\mathbf{u}^{h}\right)(0)\right\|_{0}^{2}+C\left\|\left(\mathbf{u}-\mathbf{w}^{h}\right)(0)\right\|_{0}^{2} \\
& +C\left\|\left(\mathbf{u}-\mathbf{w}^{h}\right)_{t}\right\|_{L^{\infty}\left(J ; L^{2}(\Omega)^{3}\right)}^{2}+C\left\|\mathbf{u}-\mathbf{w}^{h}\right\|_{L^{\infty}\left(J ; L^{2}(\Omega)^{3}\right)^{2}}^{2}
\end{aligned}
$$


We use this estimate in (5.37) and obtain

$$
\begin{aligned}
\|\mathbf{e}\|_{L^{\infty}\left(J ; L^{2}(\Omega)^{3}\right)}^{2} \leqslant & C\left\|\mathbf{u}_{0}-\Pi_{h} \mathbf{u}_{0}\right\|_{0}^{2}+C\left\|\left(\mathbf{u}-\mathbf{w}^{h}\right)(0)\right\|_{0}^{2} \\
& +C\left\|\left(\mathbf{u}-\mathbf{w}^{h}\right)_{t}\right\|_{L^{\infty}\left(J ; L^{2}(\Omega)^{3}\right)}^{2}+C\left\|\mathbf{u}-\mathbf{w}^{h}\right\|_{L^{\infty}\left(J ; L^{2}(\Omega)^{3}\right)}^{2} .
\end{aligned}
$$

From the approximation properties in Lemma 5.4 and standard results for the $L^{2}$-projection, we conclude that

$$
\begin{aligned}
\|\mathbf{e}\|_{L^{\infty}\left(J ; L^{2}(\Omega)^{3}\right)}^{2} \leqslant & C h^{2 \min \left\{s+\sigma_{E}, \ell+1\right\}}\left\|\mathbf{u}_{0}\right\|_{s+\sigma_{E}}^{2} \\
& +C h^{2\left(\min \{s, \ell\}+\sigma_{E}\right)}\left(\left\|\mathbf{u}_{0}\right\|_{s+\sigma_{E}}^{2}+\left\|\nabla \times \mathbf{u}_{0}\right\|_{s}^{2}\right) \\
& +C h^{2\left(\min \{s, \ell\}+\sigma_{E}\right)}\left(\left\|\mathbf{u}_{t}\right\|_{L^{\infty}\left(J ; H^{\mathrm{s}+\sigma_{E}}(\Omega)^{3}\right)}^{2}+\left\|\nabla \times \mathbf{u}_{t}\right\|_{L^{\infty}\left(J ; H^{\mathrm{s}}(\Omega)^{3}\right)}^{2}\right) \\
& +C h^{2\left(\min \{s, \ell\}+\sigma_{E}\right)}\left(\|\mathbf{u}\|_{L^{\infty}\left(J ; H^{\mathrm{s}+\sigma_{E}}(\Omega)^{3}\right)}^{2}+\|\nabla \times \mathbf{u}\|_{L^{\infty}\left(J ; H^{\mathrm{s}}(\Omega)^{3}\right)}^{2}\right) .
\end{aligned}
$$

As in fact $\mathbf{u} \in C^{0}\left(J ; H^{\mathrm{s}+\sigma_{E}}(\Omega)^{3}\right)$ and $\nabla \times \mathbf{u} \in C^{0}\left(J ; H^{\mathrm{s}}(\Omega)^{3}\right)$, we can absorb the three terms involving $\mathbf{u}_{0}$ and $\nabla \times \mathbf{u}_{0}$ into the last two. Finally, since $\min \left\{s+\sigma_{E}, \ell+1\right\} \geqslant \min \{s, \ell\}+\sigma_{E}$, the proof of Theorem 4.3 follows.

\section{Numerical experiments}

In this section, we present a series of numerical experiment to validate the error bounds from Theorems 4.1, 4.2 and 4.3. Then, we demonstrate the versatility of the DG method for the propagation of timedependent electromagnetic waves in complex media or geometry.

In all our tests, we consider the $2 \mathrm{D}$ version of our model problem. In this case, $2 \mathrm{D}$ vector fields $\mathbf{u}\left(x_{1}, x_{2}\right)=\left(u_{1}\left(x_{1}, x_{2}\right), u_{2}\left(x_{1}, x_{2}\right)\right)$ in $\mathbb{R}^{2}$ are identified with their $3 \mathrm{D}$ extensions $\mathbf{u}\left(x_{1}, x_{2}, x_{3}\right)=$ $\left(u_{1}\left(x_{1}, x_{2}, 0\right), u_{2}\left(x_{1}, x_{2}, 0\right), 0\right)$ in $\mathbb{R}^{3}$. Hence, we deduce that the curl-curl operator in (2.1) is given by

$$
\nabla \times\left(\mu^{-1} \nabla \times \mathbf{u}\right)=\left(\frac{\partial}{\partial x_{2}} \mu^{-1}\left(\frac{\partial u_{2}}{\partial x_{1}}-\frac{\partial u_{1}}{\partial x_{2}}\right),-\frac{\partial}{\partial x_{1}} \mu^{-1}\left(\frac{\partial u_{2}}{\partial x_{1}}-\frac{\partial u_{1}}{\partial x_{2}}\right)\right) .
$$

On the boundary, we have $\mathbf{n} \times \mathbf{u}=\mathbf{u} \cdot \mathbf{t}$, where $\mathbf{t}$ is the counterclockwise-oriented tangential unit vector; i.e. if $\mathbf{n}=\left(n_{1}, n_{2}\right)$, then $\left(t_{1}, t_{2}\right)=\left(-n_{2}, n_{1}\right)$. Similarly, the tangential jumps are now scalar quantities defined as $\llbracket \mathbf{u} \rrbracket_{\mathrm{T}}=\mathbf{u}^{+} \cdot \mathbf{t}^{+}+\mathbf{u}^{-} \cdot \mathbf{t}^{-}$.

The 2D version of the interior penalty method is then obtained straightforwardly, and all our theoretical results hold true in this case as well.

\subsection{Time discretization}

The DG discretization of the (2D) model problem leads to a finite system of linear second-order ordinary differential equations of the form

$$
\mathbf{M}(\varepsilon) \underline{u}^{\prime \prime}(t)+\mathbf{M}(\sigma) \underline{u}^{\prime}(t)+\mathbf{A} \underline{u}(t)=\underline{f}(t), \quad t \in \bar{J},
$$

with initial conditions

$$
\mathbf{M} \underline{u}(0)=\underline{u}_{0}, \quad \mathbf{M} \underline{u}^{\prime}(0)=\underline{v}_{0} .
$$

Here, $\underline{u}(t)$ is the coefficient vector of the finite-element approximation $\mathbf{u}^{h}(t)$ with respect to a basis of $\mathbf{V}^{h}, \underline{u}_{0}$ and $\underline{v}_{0}$ are the coefficient vectors of the discrete initial data and $\underline{f}(t)$ is the load vector at time $t$. 
The matrix A denotes the stiffness matrix associated with the DG form $a_{h}$; for $\alpha \geqslant \alpha_{\min }$, it is symmetric positive semidefinite (see Lemma 3.1). The matrices $\mathbf{M}(\varepsilon)$ and $\mathbf{M}(\sigma)$ are the mass matrices, weighted with $\varepsilon$ or $\sigma$, that are related to the time derivatives. We also set $\mathbf{M}=\mathbf{M}(1)$. The two mass matrices $\mathbf{M}$ and $\mathbf{M}(\varepsilon)$ are symmetric positive definite.

To obtain a fully discrete approximation of (6.1) and (6.2) over the time interval $J=(0, T)$, we consider a time-stepping scheme with time step $k=T / N$. Let $\underline{u}_{n}$ denote the temporal approximation to $\underline{u}\left(t_{n}\right)$ at time $t_{n}=n k, n=0,1, \ldots, N$. In the computations below, we shall consider two different time integrators depending on the electromagnetic properties of the medium. If the medium in $\Omega$ is nonconducting, i.e. $\sigma=0$ everywhere and $\mathbf{M}(\sigma)=\mathbf{0}$, we choose the second-order explicit Newmark scheme (see, e.g. Sections 8.5-8.7 in Raviart \& Thomas, 1983), which corresponds to the leap-frog scheme

$$
\begin{aligned}
\mathbf{M}(\varepsilon) \underline{u}_{1} & =\left(\mathbf{M}(\varepsilon)-\frac{k^{2}}{2} \mathbf{A}\right) \underline{u}_{0}+k \mathbf{M}(\varepsilon) \underline{v}_{0}+\frac{k^{2}}{2} \underline{f}_{0}, \\
\mathbf{M}(\varepsilon) \underline{u}_{n+1} & =\left(2 \mathbf{M}(\varepsilon)-k^{2} \mathbf{A}\right) \underline{u}_{n}-\mathbf{M}(\varepsilon) \underline{u}_{n-1}+k^{2} \underline{f}_{n}, \quad n=1, \ldots, N-1,
\end{aligned}
$$

with $\underline{f}_{n}=\underline{f}\left(t_{n}\right)$. If $\Omega$ contains a conducting region, i.e. $\sigma>0$ in parts of $\Omega$ and $\mathbf{M}(\sigma) \neq \mathbf{0}$, we instead opt for the standard explicit fourth-order RK method.

The DG mass matrix $\mathbf{M}(\varepsilon)$ is block diagonal, with block size equal to the number of degrees of freedom per element. In contrast to standard conforming edge-element discretizations (without mass lumping), we can therefore invert $\mathbf{M}(\varepsilon)$ blockwise during the assembly process. Thus, no solution of any large linear system is required during time integration and the time marching scheme remains truly explicit. We have implemented the 2D version of the DG method (3.4), augmented by the above two explicit time marching schemes, both in Matlab and in the $\mathrm{C}++$ finite-element library deal.II ${ }^{1}$ (see Bangerth et al., 2005, 2007).

\subsection{Example 1: smooth solution, quadrilateral mesh}

We let $\Omega=(0,1) \times(0,1), J=(0,0.5)$, and choose homogeneous material parameters $\varepsilon=\mu=1$. We consider two separate situations: an insulator with $\sigma=0$ and a conductor with $\sigma=1$. The initial and source data are chosen so that the solution of the $2 \mathrm{D}$ version of (2.1) is the smooth function

$$
\mathbf{u}\left(x_{1}, x_{2}, t\right)=\frac{t^{2}}{2}\left(\begin{array}{c}
\cos \left(\pi x_{1}\right) \sin \left(\pi x_{2}\right) \\
-\sin \left(\pi x_{1}\right) \cos \left(\pi x_{2}\right)
\end{array}\right) \text {. }
$$

It satisfies $\mathbf{u} \cdot \mathbf{t}=0$ on $\Gamma$. We discretize this problem on a sequence $\left\{\mathscr{T}_{h_{i}}\right\}_{i \geqslant 1}$ of square meshes of size $h_{i}=2^{-i}$ using the polynomial spaces $\mathscr{Q}^{\ell}(K), \ell=1,2,3$, with fixed stabilization $\alpha=30$. We remark that $\alpha_{\min }$ generally increases with $\ell$; for $\ell=1,2$, a smaller value of $\alpha$ is sufficient for stability.

The time step $k_{i}=h_{i} / 20$ proved to be sufficient for numerical stability for all meshes $\left\{\mathscr{T}_{h}\right\}$ and for all $\ell \leqslant 3$. In Fig. 1 , we display the relative errors in the energy and the $L^{2}$-norm at time $T=0.5$. Because the two time-stepping methods are (at least) second-order accurate, the time integration of

\footnotetext{
${ }^{1}$ See www.dealii.org.
} 

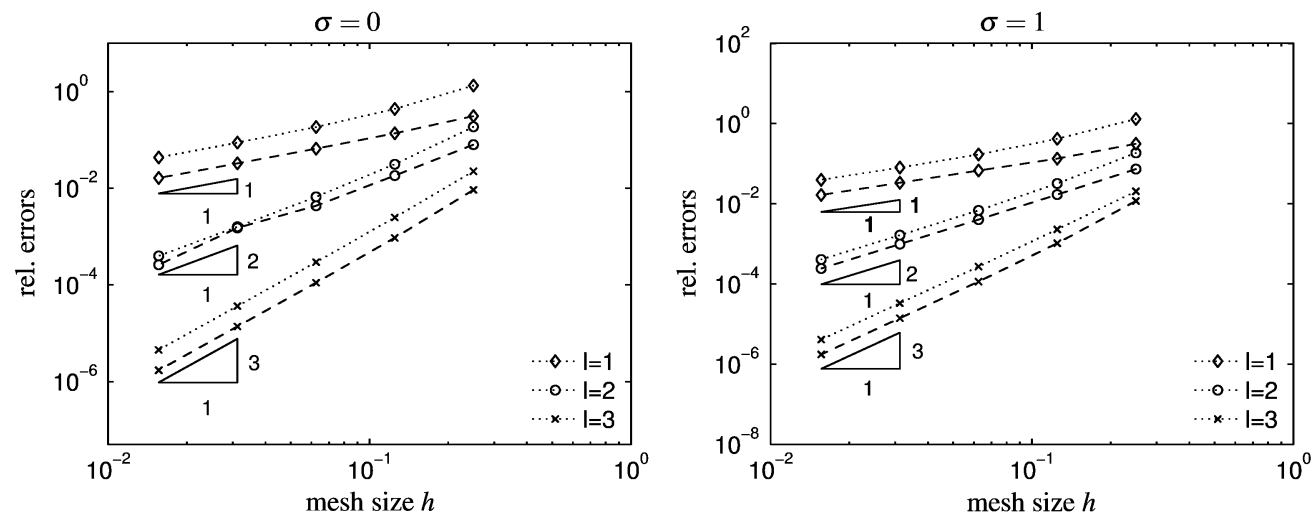

FIG. 1. Example 1: the relative errors of the DG approximation are shown at time $T=0.5$ with respect to the energy norm $(---)$ and the $L^{2}$-norm $(\cdots)$ for $\ell=1,2,3 ; \sigma=0$ (left) and $\sigma=1$ (right).

TABLE 1 Example 1: the relative errors at time $T=0.5$ in the energy norm and the $L^{2}$-norm obtained with Nédélec's second family of conforming edge elements of polynomial order $\ell=1$

\begin{tabular}{rllll}
\hline No. of elements & \multicolumn{2}{c}{ Energy error } & \multicolumn{2}{c}{$L^{2}$-error } \\
\hline 4 & $1.23 \times 10^{+01}$ & - & $5.15 \times 10^{+00}$ & - \\
16 & $1.39 \times 10^{+00}$ & 3.15 & $4.90 \times 10^{-01}$ & 3.39 \\
64 & $3.79 \times 10^{-01}$ & 1.87 & $2.76 \times 10^{-01}$ & 0.83 \\
256 & $1.04 \times 10^{-01}$ & 1.86 & $1.44 \times 10^{-01}$ & 0.94 \\
1024 & $3.30 \times 10^{-02}$ & 1.66 & $7.25 \times 10^{-02}$ & 0.98 \\
4096 & $1.30 \times 10^{-02}$ & 1.34 & $3.64 \times 10^{-02}$ & 1.00 \\
16384 & $5.98 \times 10^{-03}$ & 1.12 & $1.82 \times 10^{-02}$ & 1.00 \\
\hline
\end{tabular}

(6.4) is exact and the error consists only of the spatial error component. As the analytical solution (6.4) is arbitrarily smooth, the assumptions of Theorem 4.1 are met and we observe the predicted optimal convergence rates in the DG energy norm at time $T=0.5$.

The $L^{2}$-norm convergence rates shown in Fig. 1 are only suboptimal. We recall, however, that Theorem 4.3 only applies on simplicial meshes, so that we need to invoke Theorem 4.1 for a theoretical error bound on the error with respect to the $L^{2}$-norm, which yields the observed convergence rates of order $\mathrm{O}\left(h^{\ell}\right)$. This numerical example illustrates that the restriction to simplicial meshes in Theorem 4.3 is essential.

The same suboptimal convergence rates are obtained with conforming finite-element discretizations using full polynomial spaces $\mathscr{Q}^{\ell}(K)$ on quadrilateral or hexahedral meshes. Indeed, in Table 1 , we display the convergence rates obtained by approximating (6.4) with Nédélec's lowest order edge elements of the second kind (cf. Nédélec, 1986). As for the DG discretization in Fig. 1, the convergence rate for the energy norm (the norm in $H_{0}(\operatorname{curl} ; \Omega)$ ) is optimal, but it is suboptimal by one order for the $L^{2}$-norm. This deficiency stems from the fact that the approximation with full polynomial spaces $\mathscr{Q}^{\ell}(K)$ insufficiently separates the discrete gradients from discretely divergence-free functions (see, e.g. Section 8.2.3 in Monk, 2003). 


\subsection{Example 2: smooth solution, triangular mesh}

To demonstrate the optimal convergence of the DG method with respect to the $L^{2}$-norm, we now consider the DG discretization of the $2 \mathrm{D}$ version of $(2.1)$ on a sequence of triangular meshes with $\mathscr{P}^{1}(K)$ elements, here with $\alpha=10$. The computational domain is $\Omega=(0,1) \times(0,1)$ and the material parameters $\varepsilon=\mu=1$ and $\sigma=0$. We choose the initial conditions and the source term to match the smooth solution

$$
\mathbf{u}\left(x_{1}, x_{2}, t\right)=\cos (t)\left[\begin{array}{c}
-\mathrm{e}^{x_{1}}\left(x_{2} \cos \left(x_{2}\right)+\sin \left(x_{2}\right)\right) \\
\mathrm{e}^{x_{1}} x_{2} \sin \left(x_{2}\right)
\end{array}\right] .
$$

Since the tangential part of $\mathbf{u}$ is inhomogeneous at the boundary of $\Omega$, we need to impose inhomogeneous Dirichlet condition $\mathbf{n} \cdot \mathbf{t}=g$ on $\partial \Omega$ within our DG discretization, where $g$ is the boundary data (which is scalar in the 2D setting) and $\mathbf{t}$ is the counterclockwise-oriented tangential unit vector on $\Gamma$. To do so, we modify the (2D) semidiscrete formulation as follows: find $\mathbf{u}^{h}(t, \cdot): J \rightarrow \mathbf{V}^{h}$ such that

$$
\left(\mathbf{u}_{t t}^{h}, \mathbf{v}\right)+a_{h}\left(\mathbf{u}^{h}, \mathbf{v}\right)=(\mathbf{f}, \mathbf{v})+\sum_{E \in \mathscr{E}_{h} \mathscr{B}} \int_{E} g\left(\mathrm{a}(\mathbf{v} \cdot \mathbf{t})-\mu^{-1} \nabla \times \mathbf{v}\right) \mathrm{d} s
$$

for all $\mathbf{v} \in \mathbf{V}^{h}$ (see also Houston et al., 2004). Here, $\mathscr{E}_{h} \mathscr{B}$ is the set of all boundary edges in the underlying mesh.

We use the leap-frog scheme in time to approximate the semidiscrete solution up to $T=2 \pi$. Here, setting the time step $k_{i}=h_{i} / 4$, where $h_{i}$ denotes the smallest element diameter in the mesh, proved to be sufficient for numerical stability. In Fig. 2, we display the maximal (absolute) errors in the $L^{2}$-norm over the time interval $J=(0,2 \pi)$, which includes here both the space and time discretization errors. We observe global second-order convergence both in space and in time of the fully discrete scheme, as predicted by Theorem 4.3 for the spatial error.

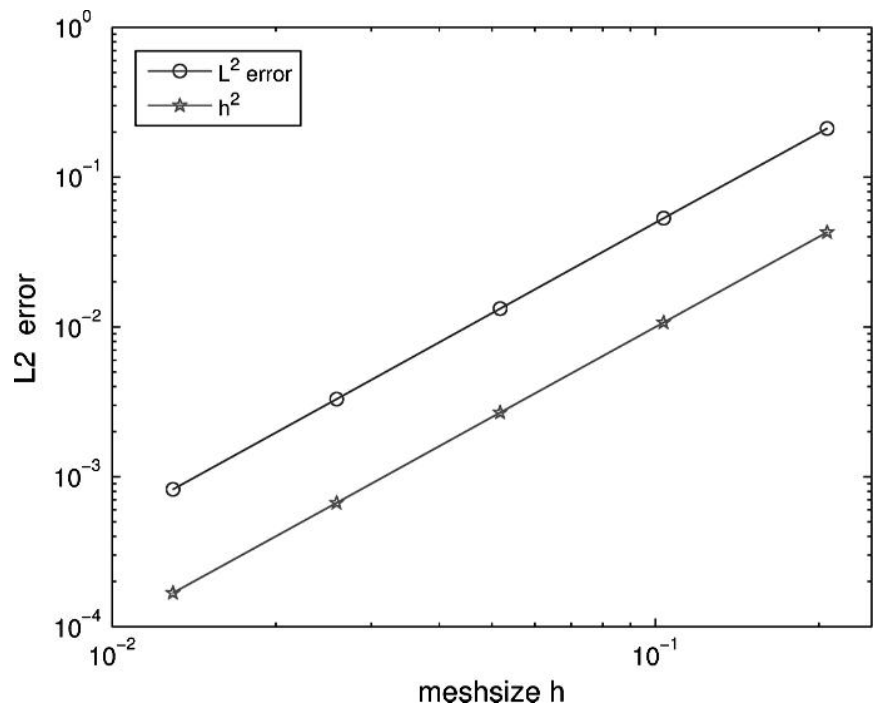

FIG. 2. Example 2: the maximal error over $0<t<2 \pi$ for the DG approximation with respect to the $L^{2}$-norm. 


\subsection{Example 3: singular solution}

To validate the error bound in Theorem 4.2, we consider an analytical solution of the 2D Maxwell's equations (2.1) on the L-shaped domain $\Omega=(-1,1)^{2} \backslash(0,1)^{2}$ with low spatial regularity. We set $\mu=$ $\varepsilon=1$ and $\sigma=0$ everywhere in $\Omega$ and choose the source and initial data such that the analytical solution in polar coordinates $(r, \phi)$ is given by

$$
\mathbf{u}(r, \phi, t)=\frac{t^{2}}{2} \nabla\left(r^{2 / 3} \sin (2 / 3 \phi)\right) .
$$

The spatial part of $\mathbf{u}$ corresponds to the gradient of the strongest corner singularity of the DirichletLaplacian on $\Omega$, the function $r^{2 / 3} \sin (2 / 3 \phi)$. Hence, we have $\mathbf{u} \in C^{\infty}\left(\bar{J} ; H^{2 / 3-\delta}(\Omega)^{2}\right)$ for all $\delta>0$. We discretize (6.7) with bilinear polynomials $(\ell=1)$ on the same sequence of meshes as in Example 1 , with $\alpha=20$. For time stepping, we use the leap-frog scheme with time step $k_{i}=h_{i} / 20$, which again yields exact integration of the time dependence of the DG approximation. As the regularity assumptions in Theorem 4.2 are satisfied by the field (6.7) with $s=2 / 3$, we expect convergence rates of order $2 / 3$ in the energy norm and in the $L^{2}$-norm, as confirmed by the results in Table 2.

\subsection{Example 4: inhomogeneous medium}

Finally, we consider an electromagnetic wave propagating through the domain $\Omega$ shown in Fig. 3 . Everywhere in $\Omega$ we set $\mu=1$, whereas the electric permeability $\varepsilon$ and the conductivity $\sigma$ vary as

TABLE 2 Example 3: the relative errors at time $T=1$ in the energy norm and the $L^{2}$-norm for the $D G$ approximation of the low-regularity solution (6.6) on the L-shaped domain

\begin{tabular}{cccccc}
\hline$i$ & No. of elements & \multicolumn{2}{c}{ Energy error } & \multicolumn{2}{c}{$L^{2}$-error } \\
\hline 1 & 48 & $2.13 \times 10^{-01}$ & - & $1.72 \times 10^{-01}$ & - \\
2 & 192 & $1.32 \times 10^{-01}$ & 0.69 & $1.16 \times 10^{-01}$ & 0.57 \\
3 & 768 & $8.25 \times 10^{-02}$ & 0.68 & $7.63 \times 10^{-02}$ & 0.61 \\
4 & 3072 & $5.17 \times 10^{-02}$ & 0.67 & $4.93 \times 10^{-02}$ & 0.63 \\
5 & 12288 & $3.24 \times 10^{-02}$ & 0.67 & $3.15 \times 10^{-02}$ & 0.65 \\
\hline
\end{tabular}

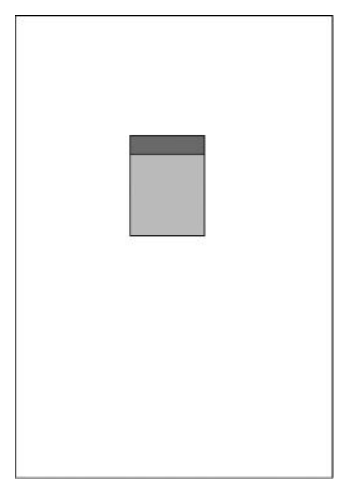

FIG. 3. Example 4: the domain $\Omega$ consists of different materials. 
follows:

$$
\varepsilon=\left\{\begin{array}{ll}
1, & \text { white region, } \\
10, & \text { light grey region, } \\
100, & \text { dark region, }
\end{array} \quad \sigma= \begin{cases}0, & \text { white and light grey region, } \\
0.3, & \text { dark grey region. }\end{cases}\right.
$$

Both the initial conditions and the source term vanish throughout $\Omega$, while the electromagnetic field is excited at the top of the domain $\Omega$ through the time-dependent (scalar) inhomogeneous boundary condition,

$$
g(x, t)=\cos (2 \pi t) \frac{1}{\sqrt{2 \pi b}} \mathrm{e}^{\frac{-x^{2}}{2 b^{2}}}, \quad b=0.2,
$$

which mimics the entry of a time-harmonic Gaussian beam. The remaining part of $\partial \Omega$ is perfectly conducting so that $\mathbf{u}$ satisfies homogeneous Dirichlet conditions there.

We discretize this problem by the 2D version of the DG method (3.4) using polynomials of degree $\ell=2$ on a fixed mesh $\mathscr{T}_{h}$ that consists of nonmatching components (generating at most one hanging node per edge), which are adapted to the discontinuities of $\varepsilon$ (recall that the wave speed in the medium is given by $(\mu \varepsilon)^{-\frac{1}{2}}$. The mesh $\mathscr{T}_{h}$ is composed of 4608 nonuniform rectangles, where the smallest local mesh size is given by $h_{\min } \approx 0.01$. The hanging nodes are naturally incorporated in the DG method without any difficulty. Compared to the uniform meshes used in the previous examples, the aspect ratio of the elements in $\mathscr{T}_{h}$ has deteriorated, which requires the larger value $\alpha=50$ with a time step $k=0.15 \cdot h_{\min }$ for stability.

In Fig. 4, the intensity of the electric field, $|\mathbf{u}|=\sqrt{\left(u_{1}^{2}+u_{2}^{2}\right)}$, is shown at times $t=2,3.5,5$ and 7 . At time $t=2$, the time-harmonic Gaussian beam excited at the top of the computational domain $\Omega$ has already impinged upon the slowest part of the scatterer (dark grey region) shown in Fig. 3, while strong field intensities appear at the corners of the interface between the two media. Then, at $t=3.5$, the wave front penetrates from the side of the lower part of the scatterer with medium wave speed (light grey region in Fig. 3). At time $t=5$, the wave front propagating downwards through the upper (slowest) part has reached the lower (somewhat faster) part of the scatterer, which leads to interference patterns beyond $t=5$.

\section{Concluding remarks}

We have presented and analysed the symmetric interior penalty DG method for the space discretization of the time-dependent Maxwell's equations in second-order form. The interior penalty DG method yields optimal a priori error bounds in the energy norm either for smooth solutions on arbitrary meshes (Theorem 4.1) or for singular solution on conforming meshes (Theorem 4.2) (see Grote et al., 2007). On conforming triangular or tetrahedral meshes, we have derived new and optimal a priori error bounds of the semidiscrete DG formulation with respect to the $L^{2}$-norm in space (Theorem 4.3). Our numerical results validate these optimal a priori estimates, both on triangular and on quadrilateral meshes. In fact, on quadrilateral meshes where Theorem 4.3 does not apply, we obtain the same suboptimal convergence rates with the DG method as with a conforming edge-element discretization using Nédélec's second family of curl-conforming elements.

When the electromagnetic field is divergence-free, the DG solution will be discretely divergencefree in the sense of Lemma 5.1. In fact, for divergence-free initial data and with $\mathbf{f}=\mathbf{0}$, the DG solution 

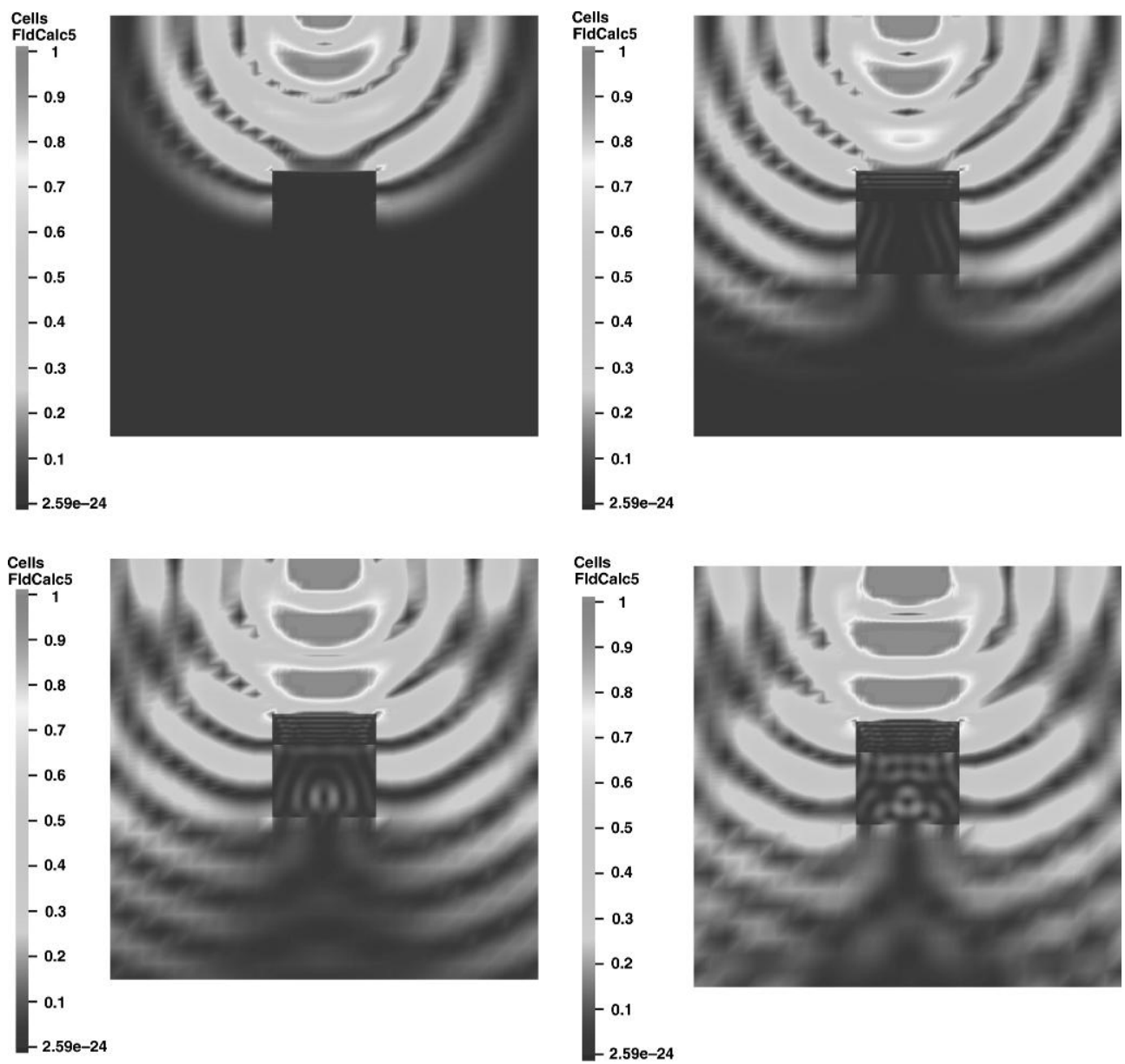

FIG. 4. Example 4: time-harmonic Gaussian beam entering the domain from above and impinging upon the scatterer displayed in Fig. 3. The intensity of the DG solution is shown at times $t=2,3.5$ (upper row) and $t=5,7$ (lower row).

will remain discretely divergence-free for all time. To obtain a globally divergence-free solution, one can use locally divergence-free polynomial spaces and then project the corresponding DG solution onto its globally divergence-free subspace as proposed in Cockburn et al. (2004).

Based on discontinuous finite-element spaces, the proposed DG method easily handles elements of various types and shapes, irregular nonmatching grids and even locally varying polynomial order. As continuity is only weakly enforced across mesh interfaces, domain decomposition techniques are straightforward. Since the resulting mass matrix is essentially diagonal, the method is inherently parallel and leads to truly explicit methods when coupled with explicit time integration. Moreover, as the stiffness matrix is symmetric positive definite, the interior penalty DG method shares the following important property with the standard continuous Galerkin approach: the semidiscrete formulation conserves (a discrete version of) the energy for all time. Therefore, if a judicious (time reversible or symplectic) time integrator such as the leap-frog scheme is used, the fully discrete scheme will also conserve (a discrete version of) the energy. 
Finally, we mention that our results are equally valid if the LDG method is used instead of the symmetric interior penalty approach presented here. For details, we refer the reader to Perugia \& Schötzau (2003).

\section{Funding}

Swiss National Science Foundation to A. S.; National Science and Engineering Council of Canada to D. S.

\section{REFERENCES}

Amrouche, C., Bernardi, C., Dauge, M. \& Girault, V. (1998) Vector potentials in three-dimensional non-smooth domains. Math. Models Appl. Sci., 21, 823-864.

Arnold, D. N., Brezzi, F., Cockburn, B. \& Marini, L. D. (2001) Unified analysis of discontinuous Galerkin methods for elliptic problems. SIAM J. Numer. Anal., 39, 1749-1779.

BAKER, G. A. (1976) Error estimates for finite element methods for second-order hyperbolic equations. SIAM J. Numer. Anal., 13, 564-576.

Bangerth, W., Hartmann, R. \& Kanschat, G. (2005) deal.II Differential Equations Analysis Library, Technical Reference, 5.2 edn. Available at:http://www.dealii.org

Bangerth, W., HARTMAnN, R. \& Kanschat, G. (2007) deal.II-a general purpose object oriented finite element library. ACM Trans. Math. Softw., 33.

Buffa, A. \& Perugia, I. (2006) Discontinuous Galerkin approximation of the Maxwell eigenproblem. SIAM J. Numer. Anal., 44, 2198-2226.

CAngellaris, A. C. \& Wright, D. B. (1991) Analysis of the numerical error caused by the stair-stepped approximation of a conducting boundary in FDTD simulations of electromagnetic phenomena. IEEE Trans. Antennas Propag., 39, 1518-1525.

Chen, M.-H., CockBURn, B. \& REITICH, F. (2005) High-order RKDG methods for computational electromagnetics. J. Sci. Comput., 22, 205-226.

Chung, E. T. \& EngQuist, B. (2006) Optimal discontinuous Galerkin methods for wave propagation. SIAM J. Numer. Anal., 44, 2131-2158.

Ciarlet, P. (1978) The Finite Element Method for Elliptic Problems. Amsterdam: North-Holland.

Cockburn, B., Li, F. \& SHU, C.-W. (2004) Locally divergence-free discontinuous Galerkin methods for the Maxwell equations. J. Comput. Phys., 194, 588-610.

Cockburn, B. \& ShU, C.-W. (1989) TVB Runge-Kutta local projection discontinuous Galerkin method for conservation laws II: general framework. Math. Comput., 52, 411-435.

Cockburn, B. \& ShU, C.-W. (2001) Runge-Kutta discontinuous Galerkin methods for convection-dominated problems. J. Sci. Comput., 16, 173-261.

ElmKies, A. \& Joly, P. (1997) Finite elements and mass lumping for Maxwell's equations: the 2D case. $C . R$. Acad. Sci. Paris Sér. I, 324, 1287-1293.

Fezoui, L., Lanteri, S., Lohrengel, S. \& Piperno, S. (2005) Convergence and stability of a discontinuous Galerkin time-domain method for the 3D heterogeneous Maxwell equations on unstructured meshes. Modél. Math. Anal. Numér., 39, 1149-1176.

Grote, M., Schneebeli, A. \& Schötzau, D. (2006) Discontinuous Galerkin finite element method for the wave equation. SIAM J. Numer. Anal., 44, 2408-2431.

Grote, M., Schneebeli, A. \& SchötZAu, D. (2007) Interior penalty discontinuous Galerkin method for Maxwell's equations: energy norm error estimates. J. Comput. Appl. Math., 204, 375-386.

Hesthaven, J. \& Warburton, T. (2002) Nodal high-order methods on unstructured grids I: time-domain solution of Maxwell's equations. J. Comput. Phys., 181, 186-221. 
HesthaVen, J. S. \& WARBURTOn, T. (2004) High-order nodal discontinuous Galerkin methods for the Maxwell's eigenvalue problem. Philos. Trans. R. Soc. Lond. Ser. A, 362, 493-524.

Hiptmair, R. (2002) Finite elements in computational electromagnetism. Acta Numer., 11, 237-339.

Houston, P., Perugia, I., Schneebeli, A. \& SchötZau, D. (2005) Interior penalty method for the indefinite time-harmonic Maxwell equations. Numer. Math., 100, 485-518.

Houston, P., Perugia, I. \& Schötzau, D. (2004) Mixed discontinuous Galerkin approximation of the Maxwell operator. SIAM J. Numer. Anal., 42, 434-459.

JiANG, B., WU, J. \& PovinELli, L. A. (1996) The origin of spurious solutions in computational electromagnetics. J. Comput. Phys., 125, 104.

Kopriva, D. A., WoodrufF, S. L. \& Hussaini, M. Y. (2000) Discontinuous spectral element approximation of Maxwell's equations. Discontinuous Galerkin Methods: Theory, Computation and Applications (B. Cockburn, G. E. Karniadakis \& C.-W. Shu eds). Lecture Notes in Computer Science Engineering, vol. 11. Berlin: Springer, pp. 355-362.

LeE, R. L. \& MAdSEN, N. K. (1990) A mixed finite element formulation for Maxwell's equations in the time domain. J. Comput. Phys., 88, 284-304.

Lions, J. \& Magenes, E. (1972) Non-Homogeneous Boundary Value Problems and Applications, vol. 1. New York: Springer.

Monk, P. (1992) A comparison of three mixed methods for the time-dependent Maxwell's equations. SIAM J. Sci. Stat. Comput., 13, 1097-1122.

Monk, P. (2003) Finite Element Methods for Maxwell's Equations. New York: Oxford University Press.

NÉDÉLEC, J. (1980) Mixed finite elements in $\mathbb{R}^{3}$. Numer. Math., 35, 315-341.

NÉDÉLEC, J. (1986) A new family of mixed finite elements in $\mathbb{R}^{3}$. Numer. Math., 50, 57-81.

PAUlsen, K. D. \& LYNCH, D. R. (1991) Elimination of vector parasities in finite element Maxwell solutions. IEEE Trans. Microw. Theory Tech., 39, 395-404.

Perugia, I. \& SchötzaU, D. (2003) The $h p$-local discontinuous Galerkin method for low-frequency timeharmonic Maxwell equations. Math. Comput., 72, 1179-1214.

Raviart, P. A. \& Thomas, J.-M. (1983) Introduction à l'Analyse Numérique des Équations aux Dérivées Partielles. Paris: Masson.

RIVIÈRE, B. \& WHEELER, M. (2001) Discontinuous finite element methods for acoustic and elastic wave problems I: semidiscrete error estimates. Technical Report 01-02. Austin, TX: TICAM, University of Texas.

Rivière, B. \& WHEELER, M. (2003) Discontinuous finite element methods for acoustic and elastic wave problems. ICM2002-Beijing Satellite Conference on Scientific Computing. Contemporary Mathematics, vol. 329. Providence, RI: AMS, pp. 271-282.

TAflove, A. (1995) Computational Electrodynamics: The Finite-Difference Time-Domain Method. Boston, MA: Artech House.

Warburton, T. (2000) Application of the discontinuous Galerkin method to Maxwell's equations using unstructured polymorphic $h p$-finite elements. Discontinuous Galerkin Methods: Theory, Computation and Applications (B. Cockburn, G. E. Karniadakis \& C.-W. Shu eds). Lecture Notes in Computer Science Engineering, vol. 11. Berlin: Springer, pp. 451-458.

Warburton, T. \& EMbree, M. (2006) The role of the penalty in the local discontinuous Galerkin method for Maxwell's eigenvalue problem. Comput. Methods Appl. Mech. Eng., 195, 3205-3323.

YEE, K. (1966) Numerical solution of initial boundary value problems involving Maxwell's equations in isotropic media. IEEE Trans. Antennas Propag., 14, 302-307. 